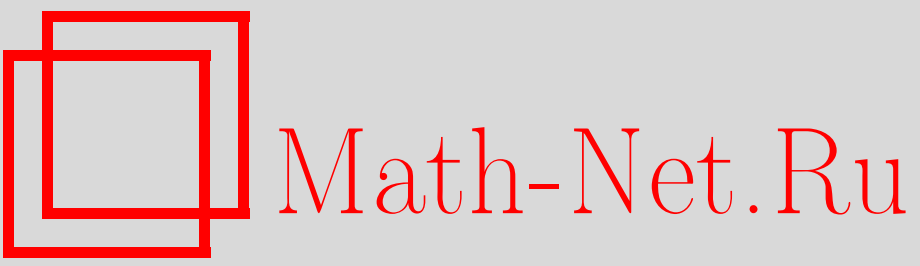

Г. М. Жислин, Спектральные свойства гамильтонианов с магнитным полем при фиксации псевдомомента. II, ТМФ, 1999, том 118, номер 1, 15-39

DOI: https://doi.org/10.4213/tmf683

Использование Общероссийского математического портала Math-Net.Ru подразумевает, что вы прочитали и согласны с пользовательским соглашением

http://www.mathnet.ru/rus/agreement

Параметры загрузки:

IP: 54.198 .67 .100

26 апреля 2023 г., 16:51:02 
ТЕОРЕТИЧЕСКАЯ

И МАТЕМАТИЧЕСКАЯ

ФИЗИКА

Том 118, № 1

январь, 1999

(C) 1999 г.

Г.М. Жислин*

\section{СПЕКТРАЛЬНЫЕ СВОЙСТВА ГАМИЛЬТОНИАНОВ С МАГНИТНЫМ ПОЛЕМ ПРИ ФИКСАЦИИ ПСЕВДОМОМЕНТА. ІІ}

Изучен дискретный спектр многочастичных гамильтонианов $H_{0}$ нейтральных систем в однородном магнитном поле при фиксации псевдомомента. Установлена общая теорема, описывающая при некоторых условиях дискретный спектр оператора $H_{0}$ в терминах свойств дискретного спектра построенных эффективных одномерных дифференциальных операторов, структура спектра которых известна. На этой основе получены условия конечности и бесконечности дискретного спектра и спектральные асимптотики оператора $H_{0}$. Результаты работы применимы к гамильтонианам любых атомов. Работа продолжает исследование, начатое в [1].

\section{ВВЕДЕНИЕ}

В настояшей работе продолжено начатое в [1] изучение спектра многочастичных гамильтонианов $H_{0}$ нейтральных систем в однородном магнитном поле после фиксации псевдомомента и отделения движения центра масс в направлении поля. Здесь мы начинаем исследование с найденного в [1] оператора $H_{0}$ (см. (1.1)) и с установленной там теоремы о локализации сушественного спектра. Главная цель данной работы - получить информацию о дискретном спектре оператора $H_{0}$, ибо ранее дискретный спектр был изучен только для системы типа атома водорода [1].

Как и в большинстве работ о дискретном спектре, мы рассматриваем здесь ситуацию, когда граница дискретного (и сушественного) спектра оператора $H_{0}$ определяется только распадениями $Z_{2}=\left\{C_{1}, C_{2}\right\}$ исходной системы ${ }^{1)}$ на две устойчивые подсистемы, т.е. когда величина inf $H_{03}\left(Z_{2}\right)$ (см. (1.4)) есть точка дискретного спектра этого оператора. При выполнении этого условия в работе впервые получены достаточные условия

\footnotetext{
1) Мы называем систему распавшейся на подсистемы $C_{1}, C_{2}$, если ее гамильтониан не содержит потенциалов взаимодействия между частицами из разных подсистем. В то же время разности координат $x_{i}-x_{j}, y_{i}-y_{j}, i \in C_{1}, j \in C_{2}$, могут входить в кинетическую часть гамильтониана.
}

\footnotetext{
*Научно-исследовательский радиофизический институт Министерства общего и профессионального образования Российской Федерации, Нижний Новгород, Россия
} 
конечности и бесконечности дискретного спектра (теоремы $1.2,1.3$ ) и - в ситуации когда дискретный спектр бесконечен - спектральные асимптотики (теорема 1.4).

Чтобы определить область применимости этих результатов, нами предпринято изучение спектральных свойств операторов $H_{03}\left(Z_{2}\right)$, отвечающих распавшейся системе. Показано, что если кластеры $C_{1}, C_{2}$ в $Z_{2}$ не нейтральны, то граница сушественного спектра оператора $H_{03}\left(Z_{2}\right)$ есть inf $H_{03}\left(Z_{3}^{\prime}\right)$ для некоторого разбиения $Z_{3}^{\prime}=\left\{C_{1}^{\prime}, C_{2}^{\prime}, C_{3}^{\prime}\right\}$, получающегося из $Z_{2}$ дроблением какой-либо из подсистем $C_{1}, C_{2}$ (теорема 1.5). Отсюда следует, что условие применимости теорем 1.2-1.4 есть неравенство

$$
\inf H_{03}\left(Z_{2}\right)<\inf H_{03}\left(Z_{3}^{\prime}\right) .
$$

Для его проверки надо исследовать природу нижней точки спектра операторов $H_{03}\left(Z_{2}\right)$, $H_{03}\left(Z_{3}^{\prime}\right)$. С этой целью для $n$-электронных атомов мы устанавливаем некоторое достаточное условие, обеспечивающее выполнение неравенства (1) (теорема 1.6). С его помошью нам удалось доказать это неравенство для $n=2,3$. Таким образом для трехчастичных систем, включая атом гелия, впервые найдены спектральные асимптотики (двухчастичные системы были изучены ранее в [1]).

Когда данная статья готовилась к печати, справедливость условия типа (1) была доказана [2] с применением теорем типа 1.5, 1.6 для произвольных атомов. Следовательно, наши обшие результаты (теоремы $1.2,1.4$ ) применимы к любым атомам, для которых таким образом получены спектральные асимптотики.

В заключение отметим, что главные результаты статьи - теоремы 1.2, 1.5, 1.6 - установлены путем дальнейшего развития геометрических методов с применением более сложных, чем ранее, типов разбиения конфигурационного пространства и что все утверждения работы учитывают перестановочную симметрию системы.

\section{1. ОПРЕДЕЛЕНИЯ И ОСНОВНЫЕ РЕЗУЛЬТАТЫ}

1.1. Мы рассматриваем нейтральную квантовую систему $Z_{1}=\{1,2, \ldots, n\} \quad n$ частищ в однородном магнитном поле, направление которого принято за направление оси $z$. Пусть $m_{i}, e_{i}, r_{i}=\left(x_{i}, y_{i}, z_{i}\right)$ - масса, заряд и радиус-вектор частицы с номером $i$,

$$
M=\sum_{j=1}^{n} m_{j}, \quad Q=\sum_{j=1}^{n} e_{j}=0 .
$$

Относительные координаты $j$-й частицы мы обозначаем через $q_{j}$ :

$$
q_{j}=\left(q_{j \perp}, q_{j 3}\right)=\left(q_{j 1}, q_{j 2}, q_{j 3}\right)=r_{j}-\sum_{t=1}^{n} m_{t} r_{t} \cdot M^{-1} .
$$

Тогда оператор энергии системы $Z_{1}$ после отделения движения центра масс в направлении 3-й оси и после сужения на подпространтво функций с фиксированными значениями $\nu_{1}, \nu_{2}$ компонент псевдомомента может быть записан в следующей форме [1]:

$$
H_{0}=T_{\perp}^{0}+T_{3}^{0}+F+V(q)
$$


где

$$
\begin{gathered}
T_{\perp}^{0}=\sum_{t=1}^{n} m_{t} \sum_{p=1}^{2}\left(\frac{1}{i} \nabla_{t p}^{0}-\mathcal{D}_{t p}\right)^{2}, \quad T_{3}^{0}=-\sum_{t=1}^{n} m_{t}\left(\nabla_{t 3}^{0}\right)^{2}, \\
\nabla_{t p}^{0}=\frac{1}{m_{t}} \frac{\partial}{\partial q_{t p}}-\frac{1}{M} \sum_{j=1}^{n} \frac{\partial}{\partial q_{j p}}, \quad p=1,2,3 \\
\mathcal{D}_{t 1}=\nu_{1}\left(\frac{1}{M}-\frac{1}{n m_{t}}\right)+B \sum_{j=1}^{n}\left(q_{j 2}-q_{t 2}\right)\left(\frac{e_{t}-e_{j}}{n m_{t}}+\frac{2 e_{j}}{M}\right), \\
\mathcal{D}_{t 2}=\nu_{2}\left(\frac{1}{M}-\frac{1}{n m_{t}}\right)-B \sum_{j=1}^{n}\left(q_{j 1}-q_{t 1}\right)\left(\frac{e_{t}-e_{j}}{n m_{t}}+\frac{2 e_{j}}{M}\right), \\
F=\left(\nu_{1}+\mathcal{E}_{2}\right)^{2}+\left(\nu_{2}-\mathcal{E}_{1}\right)^{2}, \quad \mathcal{E}_{j}=2 B \sum_{t=1}^{n} q_{t j} e_{t}, \quad V(q)=\sum_{s<t}^{1, n} V_{s t}\left(\left|q_{s}-q_{t}\right|\right) .
\end{gathered}
$$

Относительно потенциалов $V_{s t}\left(\left|q_{1}\right|\right)$ мы предполагаем, что

$$
\begin{gathered}
V_{s t}\left(\left|q_{1}\right|\right) \in \mathcal{L}_{2, \text { loc }}\left(R^{3}\right), \quad V_{s t}\left(\left|q_{1}\right|\right) \in C^{2} \text { при }\left|q_{1}\right| \neq 0, \\
V_{s t}\left(\left|q_{1}\right|\right)=e_{s} e_{t}\left|q_{1}\right|^{-\gamma} \text { при }\left|q_{1}\right|>a,
\end{gathered}
$$

где $\gamma>0, a>0$ - некоторые константы.

1.2. Пусть

$$
R_{0}=\left\{q \mid q=\left(q_{1}, \ldots, q_{n}\right), q_{i}=r_{i}-\sum_{t=1}^{n} m_{t} r_{t} \cdot M^{-1}, i=1, \ldots, n\right\}
$$

- конфигурационноепространство относительного движения системы $Z_{1}$; очевидно, при $q \in R_{0}$

$$
\sum_{j=1}^{n} m_{j} q_{j}=0 .
$$

Оператор $H_{0}$ рассматривается в пространстве $\mathcal{L}_{2}\left(R_{0}\right)$. Расширим $H_{0}$ с области $\mathcal{D}\left(H_{0}\right)=$ $C_{0}^{2}\left(R_{0}\right)$ до самосопряженного оператора, сохраняя для последнего и его области прежние обозначения $H_{0}$ и $\mathcal{D}\left(H_{0}\right)$. Пусть $S$ - группа перестановок тождественных частиц системы $Z_{1}$ и $\alpha$ - произвольный тип неприводимого представления группы ${ }^{2)} S, P^{(\alpha)}-$ проектор в $\mathcal{L}_{2}\left(R_{0}\right)$ на подпространство функций, преобразуюшихся под действием операторов $T_{g}: T_{g} \psi(q)=\psi\left(g^{-1} q\right), g \in S$, по представлению типа $\alpha, H_{0}^{(\alpha)}$ - ограничение оператора $H_{0}$ на подпространство $B^{(\alpha)}=P^{(\alpha)} \mathcal{L}_{2}\left(R_{0}\right)$.

В настоящей работе изучается дискретный спектр оператора $H_{0}^{(\alpha)}$ с областью $\mathcal{D}\left(H_{0}^{(\alpha)}\right)=P^{\alpha} \mathcal{D}\left(H_{0}\right)$.

\footnotetext{
${ }^{2)}$ Для физической значимости результатов нужно брать только те $\alpha$, которые разрешены для системы $Z_{1}$ принципом запрета Паули; в настоящей работе $\alpha$ может быть любым.
} 
1.3. Пусть $Z_{s}=\left\{C_{1}, \ldots, C_{s}\right\}$ - произвольное разбиение системы $Z_{1}$ на $s$ непересекаюшихся непустых кластеров $C_{t}$,

$$
\begin{aligned}
M\left[C_{t}\right]=\sum_{i \in C_{t}} m_{i}, \quad Q\left[C_{t}\right]=\sum_{i \in C_{t}} e_{i}, \\
R_{03}\left(Z_{s}\right)=\left\{q \mid q \in R_{0}, \sum_{k \in C_{t}} m_{k} q_{k 3}=0, t=1,2, \ldots, s\right\}, \\
R_{c 3}\left(Z_{s}\right)=\left\{q \mid q \in R_{0}, q_{j}=\left(0,0, q_{j 3}\right), j=1, \ldots, n,\right. \\
\left.\left(q, q^{\prime}\right)_{1}=0 \quad \forall q^{\prime} \in R_{03}\left(Z_{s}\right)\right\},
\end{aligned}
$$

где для любых $q=\left(q_{1}, \ldots, q_{n}\right), q^{\prime}=\left(q_{1}^{\prime}, \ldots, q_{n}^{\prime}\right)$ из $R_{0}$

$$
\left(q, q^{\prime}\right)_{1}=\sum_{j=1}^{n} m_{j}\left(q_{j}, q_{j}^{\prime}\right)_{R^{3}}
$$

Очевидно,

$$
R_{0}=R_{03}\left(Z_{s}\right) \oplus R_{c 3}\left(Z_{s}\right)
$$

$R_{03}\left(Z_{s}\right)$ и $R_{c 3}\left(Z_{s}\right)$ суть конфигурационное пространство относительного движения и пространство движения центров масс кластеров $C_{1} \ldots C_{s}$ в направлении от $z$.

Оператор энергии составной системы $Z_{s}$, состоящей из не взаимодействуюших между собой кластеров $C_{t}$, после отделения движения центра масс каждого кластера в направлении оси $z$ может быть записан в виде

$$
H_{03}\left(Z_{s}\right)=T_{\perp}^{0}+T_{3}^{0}\left(Z_{s}\right)+F+V_{Z_{s}}(q)
$$

где

$$
\begin{gathered}
T_{3}^{0}\left(Z_{s}\right)=-\sum_{t=1}^{s} \sum_{i \in C_{t}} \frac{1}{m_{t}}\left(\frac{\partial}{\partial q_{i 3}\left(Z_{s}\right)}-\frac{m_{i}}{M\left[C_{t}\right]} \sum_{p \in C_{t}} \frac{\partial}{\partial q_{p 3}}\right)^{2}, \\
V_{Z_{s}}(q)=\frac{1}{2} \sum_{t=1}^{s} \sum_{i, j \in C_{t}, i \neq j} V_{i j}\left(\left|q_{i}-q_{j}\right|\right) \\
q_{i}-q_{j}=\left(q_{i 1}-q_{j 1}, q_{i 2}-q_{j 2}, q_{i 3}\left(Z_{s}\right)-q_{j 3}\left(Z_{s}\right)\right), \\
q_{i 3}\left(Z_{s}\right)=q_{i 3}-\frac{1}{M\left[C_{t}\right]} \sum_{k \in C_{t}} m_{k} q_{k 3} \text { при } i \in C_{t} .
\end{gathered}
$$

Оператор $H_{03}\left(Z_{s}\right)$ будет рассматриваться в пространстве $\mathcal{L}_{2}\left(R_{03}\left(Z_{s}\right)\right)$. 
1.4. Пусть $\widehat{S}=\widehat{S}\left(Z_{s}\right)$ - группа перестановочной симметрии системы $Z_{s}$, порожденная группами $S\left[C_{t}\right], t=1,2, \ldots, s$, перестановок тождественных частиц в кластерах $C_{1}, \ldots, C_{s}$ и перестановками между собой тождественных кластеров $C_{i}$ из $Z_{s}$, если таковые имеются. Обозначим возможные типы неприводимых представлений группы $\widehat{S}$ в $\mathcal{L}_{2}\left(R_{0}\right), \mathcal{L}_{2}\left(R_{03}\left(Z_{s}\right)\right)$ и $\mathcal{L}_{2}\left(R_{c 3}\left(Z_{s}\right)\right)$ соответственно через $\hat{\alpha}, \hat{\alpha}_{0}$ и $\hat{\alpha}_{c}{ }^{3)}$, их матрицы через $\mathcal{D}_{g}^{(\hat{\alpha})}, \mathcal{D}_{g}^{\left(\hat{\alpha}_{0}\right)}$ и $\mathcal{D}_{g}^{\left(\hat{\alpha}_{c}\right)}$, их размерности - через $|\hat{\alpha}|,\left|\hat{\alpha}_{0}\right|$ и $\left|\hat{\alpha}_{c}\right|$. Будем писать (следуя схеме [3]), что

$\left(\hat{\alpha}_{0}, \hat{\alpha}_{c}\right) \prec \alpha$, если хотя бы одна неприводимая компонента тензорного произведения представлений $\mathcal{D}_{g}^{\left(\hat{\alpha}_{0}\right)} \otimes \mathcal{D}_{g}^{\left(\hat{\alpha}_{c}\right)}$ группы $\widehat{S}\left(Z_{s}\right)$ содержится в неприводимом представлении типа $\alpha$ группы $S$ после сужения его с $S$ на $\widehat{S}$;

$\hat{\alpha}_{0} \prec \alpha$, если найдется такой тип $\hat{\alpha}_{c}$, что $\left(\hat{\alpha}_{0}, \hat{\alpha}_{c}\right) \prec \alpha$.

Обозначим через $P^{\left(\hat{\alpha}_{0}\right)}\left(Z_{s}\right)$ проектор в $\mathcal{L}_{2}\left(R_{03}\left(Z_{s}\right)\right)$ на подпространство функций, преобразуюшихся по представлению типа $\hat{\alpha}_{0}$, и положим

$$
\begin{gathered}
H_{03}^{\left(\hat{\alpha}_{0}\right)}\left(Z_{s}\right)=H_{03}\left(Z_{s}\right) P^{\left(\hat{\alpha}_{0}\right)}, \\
H_{03}\left(\alpha ; Z_{s}\right)=\sum_{\hat{\alpha}_{0} \prec \alpha} H_{03}^{\left(\hat{\alpha}_{0}\right)}\left(Z_{s}\right)=\sum_{\hat{\alpha}_{0} \prec \alpha} H_{0}\left(Z_{s}\right) P^{\left(\hat{\alpha}_{0}\right)}, \\
\mu^{(\alpha)}=\min _{Z_{s}, s \geqslant 2} \inf H_{03}\left(\alpha ; Z_{s}\right) .
\end{gathered}
$$

Если разбиение $Z_{k}^{\prime}=\left\{C_{1}^{\prime}, \ldots, C_{k}^{\prime}\right\}$ получено из $Z_{s}$ дроблением хотя бы одного кластера из $Z_{s}$, то мы будем писать $Z_{k}^{\prime}<Z_{s}$. Так как

$$
\inf H_{03}\left(\alpha ; Z_{s}\right) \leqslant \inf H_{03}\left(\alpha ; Z_{k}^{\prime}\right) \text { при } Z_{k}^{\prime}<Z_{s},
$$

то

$$
\mu^{(\alpha)}=\min _{Z_{2}} \inf H_{03}\left(\alpha ; Z_{2}\right) .
$$

Для произвольного оператора $A$ мы обозначаем далее через $\sigma_{\mathrm{ess}}(A), \sigma_{\mathrm{d}}(A), \sigma_{\mathrm{p}}(A)$ и $\sigma_{\mathrm{pp}}(A)$ соответственно его существенный, дискретный, точечный и чисто точечный спектры.

1.5. ТЕОРема 1.1. Для любъх $\alpha$

$$
\sigma_{\mathrm{ess}}\left(H_{0}^{\alpha}\right)=\left[\mu^{\alpha} ;+\infty\right)
$$

Доказательство теоремы 1.1 дано в [1]. Мы приводим ее здесь потому, что будем неоднократно к ней обрашаться и т.к. хотим дать более простое (эквивалентное) определение соотношения $\hat{\alpha}_{0}\left(Z_{2}\right) \prec \alpha$, используемого в определении оператора $H_{03}\left(\alpha ; Z_{2}\right)$ и, значит, в выражении (1.5) для числа $\mu^{(\alpha)}$.

\footnotetext{
3) Когда мы говорим о представлении группы $S$ или ее подгрупп в каком-либо подпространстве из $\mathcal{L}_{2}\left(R_{0}\right)$, мы всегда имеем в виду представление операторами $T_{g}$ (см. п. 1.2$)$.
} 
Пусть $\mathcal{N}_{\hat{\alpha}}^{\alpha}\left(Z_{2}\right)$ - кратность представления типа $\hat{\alpha}$ группы $\widehat{S}\left(Z_{2}\right)$ в представлении типа $\alpha$ после сужения последнего с $S$ на $\widehat{S}\left(Z_{2}\right)$. Если кластеры $C_{1}, C_{2}$ не тождественны $\left(C_{1} \nsim C_{2}\right)$, то группа $\widehat{S}\left(Z_{2}\right)$ в $\mathcal{L}_{2}\left(R_{c 3}\left(Z_{2}\right)\right)$ имеет только тождественное представление, т.к. перестановки из $\widehat{S}\left(Z_{2}\right)$ не меняют координаты векторов из $R_{c 3}\left(Z_{2}\right)$. Поэтому в данном случае $\mathcal{D}_{g}^{\hat{\alpha}_{0}} \otimes \mathcal{D}_{g}^{\hat{\alpha}_{c}}=\mathcal{D}_{g}^{\hat{\alpha}_{0}}$ и соотношение $\hat{\alpha}_{0}\left(Z_{2}\right) \prec \alpha$ означает, что $\mathcal{N}_{\hat{\alpha}_{0}}^{\alpha} \geqslant 1$. Если кластеры $C_{1}$ и $C_{2}$ тождественны $\left(C_{1} \sim C_{2}\right)$, то группа $\widehat{S}\left(Z_{2}\right)$ имеет в $R_{c 3}\left(Z_{2}\right)$ два одномерных неприводимых представления, типы которых мы обозначаем через $\hat{\alpha}_{c}^{ \pm}$. В обоих представлениях перестановкам частиц внутри $C_{j}, j=1,2$, отвечает число 1 ; перестановке кластеров $C_{1} \leftrightarrow C_{2}$ в представлении типа $\hat{\alpha}_{c}^{+}$отвечает число 1 , а в представлении типа $\alpha_{c}^{-}$- число -1. Поэтому представление $\mathcal{D}_{g}^{\hat{\alpha}}:=\widehat{\mathcal{D}}_{g}^{\hat{\alpha}_{0}} \otimes \mathcal{D}_{g}^{\hat{\alpha} c}$ при $\hat{\alpha}_{c}=\hat{\alpha}_{c}^{ \pm}$неприводимо; $\hat{\alpha}=\hat{\alpha}_{0}$ при $\hat{\alpha}_{c}=\hat{\alpha}_{c}^{+}$; при $\hat{\alpha}_{c}=\hat{\alpha}_{c}^{-}$мы обозначим его тип через $-\hat{\alpha}_{0}$. Соотношение $\hat{\alpha}_{0} \prec \alpha$ в этом случае означает, что хотя бы одна из величин $\mathcal{N}_{\hat{\alpha}_{0}}^{\alpha}\left(Z_{2}\right), \mathcal{N}_{-\hat{\alpha}_{0}}^{\alpha}\left(Z_{2}\right)$ не равна нулю. Положим

$$
\begin{array}{lll}
d_{Z_{2}}\left(\alpha, \hat{\alpha}_{0}\right)=\mathcal{N}_{\hat{\alpha}_{0}}^{\alpha}\left(Z_{2}\right) & \text { при } & C_{1} \nsim C_{2}, \\
d_{Z_{2}}\left(\alpha, \hat{\alpha}_{0}\right)=\mathcal{N}_{\hat{\alpha}_{0}}^{\alpha}\left(Z_{2}\right)+\mathcal{N}_{-\hat{\alpha}_{0}}^{\alpha}\left(Z_{2}\right) & \text { при } & C_{1} \sim C_{2} .
\end{array}
$$

Тог да условие $\hat{\alpha}_{0} \prec \alpha$ эквивалентно условию $d_{Z_{2}}\left(\alpha, \hat{\alpha}_{0}\right) \geqslant 1$.

1.6. Пусть

$$
\begin{aligned}
O(\alpha) & =\left\{Z_{2} \mid \inf H_{03}\left(\alpha ; Z_{2}\right)=\mu^{(\alpha)}\right\} \\
O_{\mathrm{d}}(\alpha) & =\left\{Z_{2} \mid Z_{2} \in O(\alpha), \mu^{(\alpha)} \in \sigma_{\mathrm{d}}\left(H_{03}\left(\alpha ; Z_{2}\right)\right)\right\} .
\end{aligned}
$$

$O_{\mathrm{d}}(\alpha)$ - это множество устойчивых двухкластерных систем $Z_{2}$, нижняя грань энергии которых является нижней гранью сушественного спектра оператора $H_{0}^{(\alpha)}$. Мы будем изучать дискретный спектр оператора $H_{0}^{(\alpha)}$ в предположении, что

$$
O(\alpha)=O_{\mathrm{d}}(\alpha) \text {. }
$$

Разумеется, это условие выполняется не всегда, однако оно является обычным для работ по исследованию дискретного спектра (см., например, [3-5]). Кроме того, отметим, что в отсутствие магнитного поля равенство (1.7) выполняется, в частности, для любых атомов и положительных ионов, а также для однократных отрицательных ионов [5]. При $Z_{2} \in O_{\mathrm{d}}(\alpha)$ обозначим через $W\left(\alpha ; Z_{2}\right)$ собственное подпространство оператора $H_{03}\left(\alpha ; Z_{2}\right)$, отвечаюшее его собственному значению $\mu^{(\alpha)}$.

Кроме выполнения равенства (1.7) мы предполагаем также, что:

$A_{1}$. Все $Z_{2}^{\prime} \in O(\alpha)$ могут быть получены из одного $Z_{2} \in O(\alpha)$ перестановками тождественных частиц.

$A_{2}$. Представление группы $\widehat{S}\left(Z_{2}\right)$ в $W\left(\alpha ; Z_{2}\right)$ неприводимо, т.е. найдется такой тип $\hat{\alpha}_{0}$, что

$$
P^{\left(\hat{\alpha}_{0}\right)} W\left(\alpha ; Z_{2}\right)=W\left(\alpha ; Z_{2}\right) \quad \text { и } \quad \operatorname{dim} W\left(\alpha ; Z_{2}\right)=\left|\hat{\alpha}_{0}\right| .
$$

Условия $A_{1}, A_{2}$ носят чисто технический характер и служат для упрошения формулировок и доказательств. Мы будем указывать, какие изменения в формулировках необходимо сделать, если $A_{1}$ или $A_{2}$ не выполняется. 
1.7. Условие (1.7) подсказывает, что вклад распадения $Z_{2}=\left\{C_{1}, C_{2}\right\}$ в дискретный спектр оператора $H_{0}^{(\alpha)}$ должен зависеть от взаимодействия между кластерами $C_{1}, C_{2}$ из $Z_{2}$, когда система $Z_{2}$ находится в основном состоянии. Оценивая это взаимодействие сверху и снизу для больших значений

$$
\left|\zeta_{3}\right|=\left|\sum_{j \in C_{1}} \frac{m_{j} q_{j 3}}{M\left[C_{1}\right]}-\sum_{i \in C_{2}} \frac{m_{i} q_{i 3}}{M\left[C_{2}\right]}\right|,
$$

можно получить одномерные эффективные потенциалы

$$
\begin{array}{llll}
V_{Z_{2}}^{ \pm}\left(\zeta_{3}\right) & =Q\left(Z_{2}\right)\left|\zeta_{3}\right|^{-\gamma} \pm c_{0}\left|\zeta_{3}\right|^{-\gamma_{0}}, \quad \text { если } & \left|\zeta_{3}\right| \geqslant b_{0}, \\
V_{Z_{2}}^{+}\left(\zeta_{3}\right) & =Q\left(Z_{2}\right) b_{0}^{-\gamma}, \quad V_{Z_{2}}^{-}\left(\zeta_{3}\right) \equiv 0, & \text { если } & \left|\zeta_{3}\right|<b_{0},
\end{array}
$$

и ввести, далее, эффективные одномерные операторы в $\mathcal{L}_{2}\left(R^{1}\right)$ :

$$
h_{Z_{2}}^{ \pm}=-M\left(Z_{2}\right)^{-1} \frac{d^{2}}{d \zeta_{3}^{2}}+V_{Z_{2}}^{ \pm}\left(\zeta_{3}\right),
$$

где $b_{0}$ и $c_{0}$ - некоторые константы, $M\left(Z_{2}\right)^{-1}=M\left[C_{1}\right]^{-1}+M\left[C_{2}\right]^{-1}, Q\left(Z_{2}\right)=Q\left[C_{1}\right] \times$ $Q\left[C_{2}\right], \quad \gamma_{0}=\min (\gamma+d, 3)$. Здесь $d=2$, если выполнено условие $A_{2}$ или более общее условие

$A_{3}$. Все функции из $W\left(\alpha ; Z_{2}\right)$, отвечаюшие одному и тому же типу перестановочной симметрии, имеют одну и ту же четность относительно инверсии $q_{i 3} \rightarrow-q_{i 3}{ }^{4}$.

В остальных случаях в выражении для $\gamma_{0} d=1$.

Для $\lambda<0$ обозначим через $N\left(\lambda ; h_{Z_{2}}^{ \pm}\right)$и $N\left(\mu^{(\alpha)}+\lambda, H_{0}^{(\alpha)}\right)$ размерности линейных оболочек собственных векторов операторов $h_{Z_{2}}^{ \pm}$и $H_{0}^{(\alpha)}$, отвечающих собственным значениям этих операторов, не превосходящим соответственно $\lambda$ и $\mu^{(\alpha)}+\lambda$.

1.8. Основной результат этой работы - теорема 1.2 .

Теорема 1.2. Пусть условие (1.7) верно и $Q\left(Z_{2}\right)<0 \quad \forall Z_{2} \in O(\alpha)$. Тогда для любого типа а неприводимого представления группь $S$, всех $\lambda<0$ и некоторой константы $c^{0}$

$$
-c^{0}+d_{Z_{2}}\left(\alpha, \hat{\alpha}_{0}\right) N\left(\lambda ; h_{Z_{2}}^{+}\right) \leqslant N\left(\mu^{(\alpha)}+\lambda ; H_{0}^{(\alpha)}\right) \leqslant c^{0}+d_{Z_{2}}\left(\alpha, \hat{\alpha}_{0}\right) N\left(\lambda ; h_{Z_{2}}^{-}\right) .
$$

\footnotetext{
4) Условие $A_{2}$ является достаточным для справедливости $A_{3}$, ибо если бы $A_{3}$ не выполнялось для какого-то типа $\hat{\alpha}_{0}^{\prime}$ неприводимого представления группы $\widehat{S}\left(Z_{2}\right)$ в $W\left(\alpha ; Z_{2}\right)$, то тогда подпространство $W\left(\alpha ; Z_{2}\right)$ содержало бы не менее двух базисов представления типа $\hat{\alpha}_{0}^{\prime}$, один из которых четный, а другой нечетный по отношению к инверсии $q_{j 3} \rightarrow-q_{j 3}$, что противоречило бы требованию $\operatorname{dim} W\left(\alpha, Z_{2}\right)=\left|\hat{\alpha}_{0}^{\prime}\right|$, содержашемуся в $A_{2}$.
} 


\section{ЗАМЕЧАНИЯ.}

1. Эффективные операторы $h_{Z_{2}}^{ \pm}$не зависят явно от фиксированного значения псевдомомента $\nu=\left(\nu_{1}, \nu_{2}\right)$, однако от $\nu$ может зависеть константа $c^{0}$ в $(1.10)$. Кроме того (и это наиболее важно), от $\nu$ могут зависеть граница существенного спектра - число $\mu^{\alpha}$ и класс $O(\alpha)$, а следовательно, и эффективный потенциал. Однако сейчас мы не готовы привести какие-либо примеры.

2. Если $Q\left(Z_{2}\right)=0$, то доказательство теоремы 1.2 остается в силе, однако для получения содержательных результатов надо иметь более точные выражения для эффективных потенциалов $V_{Z_{2}}^{ \pm}\left(\zeta_{3}\right)$. Чтобы не усложнять изложение, мы не будем их искать. Кроме того, при $Q\left(Z_{2}\right) \equiv Q\left[C_{1}\right] \cdot Q\left[C_{2}\right]=0$ вьполняется равенство $Q\left[C_{1}\right]=-Q\left[C_{2}\right]=0$. В такой ситуации мы не имеем подходов к проверке условия (1.7) (см. теорему 1.5$)$ и не можем указать ни одного примера, где оно выполняется.

3. При нарушении условия $A_{1}$ множество $O(\alpha)$ разбивается на непересекаюшиеся классы $\mathcal{K}_{i}$ так, чтобы все $Z_{2}^{\prime}$ из класса $\mathcal{K}_{i}$ получались из некоторого $Z_{2 i} \in \mathcal{K}_{i}$ перестановками тождественных частищ и чтобы ни одно $Z_{2} \in \mathcal{K}_{j}$ при $j \neq i$ не обладало этим свойством. Тогда вместо (1.10) будет справедливо соотношение

$$
\begin{aligned}
-c^{0}+\sum_{i} d_{Z_{2 i}}\left(\alpha ; \hat{\alpha}_{0 i}\right) N\left(\lambda ; h_{Z_{2 i}}^{+}\right) & \leqslant N\left(\mu^{(\alpha)}+\lambda, H_{0}^{(\alpha)}\right) \leqslant \\
& \leqslant c^{0}+\sum_{i} d_{Z_{2 i}}\left(\alpha, \hat{\alpha}_{0 i}\right) N\left(\lambda ; h_{Z_{2 i}}^{-}\right),
\end{aligned}
$$

где $\hat{\alpha}_{0 i}$ определяется для разбиения $Z_{2 i}$ так же, как $\hat{\alpha}_{0}$ для $Z_{2}$. При этом мы предполагаем, что для каждого $Z_{2 i}$ условие $A_{2}$ выполняется, если там положить $Z_{2}=Z_{2 i}$, $\hat{\alpha}_{0}=\hat{\alpha}_{0 i}$.

4. Если условие $A_{2}$ не выполнено для разбиения $Z_{2}$ или для какого-то разбиения $Z_{2 i}$ (см. замечание 3$)$, то в неравенствах $(1.10),(1.11)$ вместо коэффициентов $d_{Z_{2}}\left(\alpha, \hat{\alpha}_{0}\right)$ и $d_{Z_{2 i}}\left(\alpha, \hat{\alpha}_{0 i}\right)$ надо взять соответственно

$$
d_{Z_{2}}(\alpha)=\sum_{s=1}^{s_{0}} k_{0}^{(s)} d_{Z_{2}}\left(\alpha, \hat{\alpha}_{0}^{(s)}\right) \text { и } d_{Z_{2 i}}(\alpha)=\sum_{t=1}^{t_{i}} k_{i}^{(t)} d_{Z_{2}}\left(\alpha, \hat{\alpha}_{0 i}^{(t)}\right),
$$

где $\hat{\alpha}_{0}^{(s)}$ и $\hat{\alpha}_{0 i}^{(t)}$ - все типы неприводимых представлений групп $\widehat{S}\left(Z_{2}\right)$ и $\widehat{S}\left(Z_{2 i}\right)$, для которых соответственно

$$
\begin{gathered}
P^{\left(\hat{\alpha}_{0}^{(s)}\right)} W\left(\alpha ; Z_{2}\right) \neq 0, \quad s=1, \ldots, s_{0}, \\
P^{\left(\hat{\alpha}_{0 i}^{(t)}\right)} W\left(\alpha ; Z_{2 i}\right) \neq 0, \quad t=1,2, \ldots, t_{i},
\end{gathered}
$$

$k_{0}^{(s)}$ и $k_{i}^{(t)}$-кратностипредставлений типов $\hat{\alpha}_{0}^{(s)}$ и $\alpha_{0 i}^{(t)}$ в $W\left(\alpha ; Z_{2}\right)$ и $W\left(\alpha ; Z_{2 i}\right)$. При этом в зависимости от вьполнения (или невыполнения) условия $A_{3}$ (п. 1.7) показатель $\gamma_{0}$ во втором слагаемом эффективного потенциала $(1.8)$ равен $\min (\gamma+2,3)$ или $\min (\gamma+1,3)$. 
5. Эффективные операторы $h_{Z_{2}}^{ \pm}$и формулировка теоремы 1.2 практически совпадают с эффективными операторами и теоремой 1 из [4], однако это совпадение чисто внешнее. В [4] исследуются ограничения операторов многочастичных систем на подпространства функций фиксированного веса $m S O(2)$-симметрии, в то время как здесь изучаются ограничения на подпространство состояний с фиксированным значением псевдомомента. А главное, результаты [4] вообще не применимы к нейтральным системам, ибо для таких систем использование $S O(2)$-симметрии не позволяет найти границу сушественного спектра (отсутствует аналог теоремы Хунцикера-ван Винтера-Жислина).

1.9. Используя теорему 1.2 и известные спектральные свойства операторов $h_{Z_{2}}^{ \pm}$ $[6,7]$, можно установить спектральные свойства оператора $H_{0}^{(\alpha)}$. Считаем далее, что $\alpha$ произвольно, (1.7) выполняется и $Q\left(Z_{2}\right)<0$ для всех $Z_{2} \in O(\alpha)$.

Теорема 1.3. Дискретный спектр оператора $H_{0}^{(\alpha)}$ конечен, если $\gamma>2$ или если $\gamma=2$ и $Q\left(Z_{2}\right) \geqslant-4^{-1} M\left(Z_{2}\right)^{-1}$, и бесконечен, если $\gamma=2$ и $Q\left(Z_{2}\right)<-4^{-1} M\left(Z_{2}\right)^{-1}$ или если $\gamma<2$ и $Q\left(Z_{2}\right)<0$.

TEOPEMA 1.4 .

1. При $\lambda \rightarrow-0, \quad \gamma<2$ u $Q\left(Z_{2}\right)<0$

$$
N\left(\mu^{(\alpha)}+\lambda ; H_{0}^{(\alpha)}\right)=|\lambda|^{(\gamma-2) / 2 \gamma} d\left(\alpha, \hat{\alpha}_{0}\right) M\left(Z_{2}\right)^{1 / 2}\left|Q\left(Z_{2}\right)\right|^{1 / \gamma} J(\gamma)+\mathcal{R},
$$

$2 \partial e$

$$
J(\gamma)=\gamma^{-1} \int_{1}^{\infty}(u-1)^{1 / 2} u^{-(\gamma+1) / \gamma} d u, \quad \mathcal{R}=O(|\ln | \lambda||) .
$$

2. При $\lambda \rightarrow-0, \gamma=2$ u $Q\left(Z_{2}\right)<-4^{-1} M\left(Z_{2}\right)^{-1}$

$$
N\left(\mu^{(\alpha)}+\lambda ; H_{0}^{(\alpha)}\right)=\frac{1}{2 \pi}|\ln | \lambda|| d\left(\alpha, \hat{\alpha}_{0}\right)\left(4\left|Q\left(Z_{2}\right)\right| M\left(Z_{2}\right)-1\right)^{1 / 2}+o(|\ln | \lambda||) .
$$

ЗАМЕЧАНИЯ.

1. Теоремы 1.3, 1.4 сформулированы для простоты в предположениях $A_{1}, A_{2}$. Для получения формулировок в общем случае надо использовать замечания 3,4 к теореме 1.2 и учесть, что если нарушено условие $A_{2}$ и хотя бы для одного $Z_{2 i}$ не выполняется $A_{3}$, то при $\gamma<1 \mathcal{R}=O\left(|\lambda|^{(\gamma-1) / 2 \gamma}\right)$.

2. При $\gamma=1$ (кулоновские потенциалы) $J(\gamma)=J(1)=\pi / 2$.

1.10. Полученные результаты можно применить к гамильтониану любого атома с фиксированным псевдомоментом (см. п. 1.11-1.14). Мы ограничимся здесь только случаем атома гелия, так как для него формулировка результатов достаточно проста. Пусть $Z_{1}=(1,2,3)$, где 1 - номер ядра, 2 и 3 - номера электронов, $e_{2}=e_{3}=-e_{1} / 2$, $m_{2}=m_{3}, V_{i j}\left(r_{i j}\right)=e_{i} e_{j}\left|r_{i j}\right|^{-1}$. Возможные типы симметрии $\alpha$ системы $Z_{1}$ отвечают симметричному (тождественному) и антисимметричному представлениям группы перестановок двух электронов. Для обоих типов $\alpha$

$$
\begin{gathered}
O(\alpha)=\left\{Z_{2}(2), Z_{2}(3)\right\}, \quad \text { где } Z_{2}(j)=\left\{C_{1 j}, C_{2 j}\right\}, \\
C_{1 j}=Z_{1} \backslash(j), \quad C_{2 j}=(j), \quad j=2,3 .
\end{gathered}
$$


Очевидно, $Q\left(Z_{2}(j)\right)<0$. Так как оператор $H_{03}\left(Z_{2}(j)\right)$ не обладает перестановочной симметрией, то $H_{03}\left(\alpha ; Z_{2}(j)\right)=H_{03}\left(Z_{2}(j)\right)$. В п. $1.13,1.14$ будет показано, что $\sigma_{\mathrm{d}}\left(H_{03}\left(Z_{2}(j)\right)\right) \neq \varnothing$ и, значит, (1.7) вьполняется. Используя теоремы $1.2,1.4$ и замечание 4 к теореме 1.2 , мы получаем

$$
N\left(\mu^{\alpha}+\lambda ; H_{0}^{\alpha}\right)=|\lambda|^{-1 / 2} e_{2}^{2}\left(\frac{m_{2}\left(m_{1}+m_{2}\right)}{M}\right)^{1 / 2} d(\alpha) \cdot \frac{\pi}{2}+O(|\ln | \lambda||),
$$

где $d(\alpha)=\operatorname{dim} W\left(\alpha ; Z_{2}(j)\right)$. Мы ожидаем, что $d(\alpha)=1$, но пока это не доказано.

1.11. Применимость теорем $1.2-1.4$ к конкретным системам зависит от справедливости для них соотношения (1.7). Так как проверка (1.7) должна быть основана на знании положения $\sigma_{\text {ess }}\left(H_{03}\left(\alpha ; Z_{2}\right)\right)$ для $Z_{2} \in O(\alpha)$, то далее мы устанавливаем теоремy 1.5 о локализации $\sigma_{\mathrm{ess}}\left(H_{03}\left(\alpha ; Z_{2}\right)\right)$ и на ее основе для систем типа атомов даем простое достаточное условие непустоты $\sigma_{\mathrm{d}}\left(H_{03}\left(\alpha ; Z_{2}\right)\right)$ - теорему 1.6 . Использование теорем 1.5, 1.6 позволило доказать здесь соотношение (1.7) для трехчастичных систем типа атома гелия, а в [2] и для произвольных атомов. Введем обозначение

$$
\mu\left(\alpha ; Z_{2}\right)=\min _{Z_{s}^{\prime}<Z_{2}} \inf H_{03}\left(\alpha ; Z_{s}^{\prime}\right)
$$

TEOPEMA 1.5. Пусmь $Q\left(Z_{2}\right)<0$. Тогда

$$
\sigma_{\mathrm{ess}}\left(H_{03}\left(\alpha ; Z_{2}\right)\right)=\left[\mu\left(\alpha ; Z_{2}\right),+\infty\right)
$$

ЗАмечАниЕ. Так как каждое разбиение системы $Z_{1}$ на $s$ подсистем при $s>3$ одновременно является и разбиением на 3 подсистемы, то в (1.12) мы можем брать только $Z_{s}^{\prime}=Z_{3}^{\prime}$.

1.12. Чтобы понять смысл теоремы 1.5 , сравним ситуации с магнитным полем и без него. Будем обозначать через $\widehat{\mathcal{H}}_{0}\left(Z_{s}^{\prime}\right)$ оператор энергии составной системы $Z_{s}^{\prime}=\left\{C_{1}^{\prime}, \ldots, C_{s}^{\prime}\right\}$ в отсутствие магнитного поля после отделения движения центров масс кластеров $C_{1}^{\prime} \ldots C_{s}^{\prime}$ в $R^{3}$. Тогда в ситуации без магнитного поля именно оператор $\widehat{\mathcal{H}}_{0}\left(Z_{2}\right)$ соответствует оператору $H_{03}\left(Z_{2}\right)$. Согласно [8] (см. также [9])

$$
\inf \left\{\lambda \mid \lambda \in \sigma_{\mathrm{ess}}\left(H_{03}\left(Z_{2}\right)\right)\right\}=\inf _{\left\{\psi_{p}\right\}} \underline{\lim }\left(\widehat{\mathcal{H}}_{0}\left(Z_{2}\right) \psi_{p}, \psi_{p}\right)
$$

где $\left\{\psi_{p}\right\}$ - последовательности Вейля, отвечаюшие всем возможным распадениям $Z_{2}^{\prime}=$ $\left\{C_{1}^{\prime}, C_{2}^{\prime}\right\}$ исходной системы $Z_{2}=\left\{C_{1}, C_{2}\right\}$. Если $\psi_{p}$ описывает распадение $Z_{2}^{\prime}=Z_{2}$, т.е. уход частищ кластера $C_{1}\left\{C_{2}\right\}$ от кластера $C_{2}\left\{C_{1}\right\}$, то "уходяшая на бесконечность" подсистема $C_{j}$ обязана распадаться, ибо положение ее центра масс фиксировано. Если $\psi_{p}$ описывает распадение $Z_{2}^{\prime}=\left\{C_{1}^{\prime}, C_{2}^{\prime}\right\} \neq Z_{2}$, то, т.к. взаимодействие между кластерами $C_{1}$ и $C_{2}$ отсутствует, $Z_{2}^{\prime}$ отвечает распадению на кластеры $C_{1}^{\prime} \cap C_{1}, C_{1}^{\prime} \cap C_{2}$, 
$C_{2}^{\prime} \cap C_{1}, C_{2}^{\prime} \cap C_{2}$, из которых не менее трех непустые. Таким образом, в отсутствие магнитного поля любая последовательность Вейля $\psi_{p}$ для оператора $\widehat{\mathcal{H}}_{0}\left(Z_{2}\right)$ описывает какое-то распадение $Z_{s}^{\prime}, s \geqslant 3$ и, значит,

$$
\inf \left\{\lambda \mid \lambda \in \sigma_{\text {ess }}\left(\mathcal{H}_{0}\left(Z_{2}\right)\right)\right\} \geqslant \min _{Z_{s}^{\prime}<Z_{2}} \inf \widehat{\mathcal{H}}_{0}\left(Z_{s}^{\prime}\right) .
$$

Рассмотрим теперь ситуацию с магнитным полем. Аналогично (1.14) (см. $[8,9])$

$$
\inf \left\{\lambda \mid \lambda \in \sigma_{\text {ess }}\left(H_{03}\left(Z_{2}\right)\right)\right\}=\inf _{\left\{\psi_{p}\right\}} \underline{\lim }\left(H_{03}\left(Z_{2}\right) \psi_{p}, \psi_{p}\right)
$$

где $\left\{\psi_{p}\right\}$ - последовательности Вейля для оператора $H_{03}\left(Z_{2}\right)$. Движение центра масс каждого кластера $C_{j} \in Z_{2}$ отделено лишь в направлении от $z$. Поэтому рассуждения, приведенные ранее для ситуации в отсутствие магнитного поля, применимы только к последовательностям Вейля, описываюшим или распадения $Z_{2}^{\prime} \neq Z_{2}$, или $Z_{2}^{\prime}=$ $Z_{2}$, но в направлении оси $z$. Другими словами, если $\psi_{p}$ отвечает распадению $Z_{2}^{\prime}=$ $\left\{C_{1}^{\prime}, C_{2}^{\prime}\right\} \neq Z_{2}$ или распадению $Z_{2}^{\prime}=Z_{2}$ за счет увеличения расстояний между частицами кластеров $C_{1}, C_{2}$ в направлении оси $z$, то аналогично предыдушему можно заключить, что $\psi_{p}$ описывает какое-то распадение $Z_{s}^{\prime}<Z_{2}, s \geqslant 3$. Однако если $\psi_{p}$ описывает распадение $Z_{2}^{\prime}=Z_{2}$ за счет увеличения расстояний между кластерами $C_{1}, C_{2}$ в плоскости $x, y$, то сделать такой вывод априори нельзя. Другими словами, априори мы не можем исключить, что сушественный спектр оператора $H_{03}\left(Z_{2}\right)$ возникает за счет бесконечного увеличения расстояний между кластерами $C_{1}, C_{2}$ в плоскости $x, y$. Теорема 1.5 показывает, что в случае $Q\left(Z_{2}\right)<0$ такая ситуация невозможна (вследствие фиксации псевдомомента), т.е. что при $Q\left(Z_{2}\right)<0$ граница сушественного спектра оператора $H_{0}\left(\alpha ; Z_{2}\right)$ определяется распадением не менее чем одного кластера $C_{i} \in Z_{2}$ (а не движением нераспавшихся кластеров $C_{1}, C_{2}$ в плоскости $\left.x, y\right)$. Доказательство теоремы 1.5 дано в п. 3.1-3.8.

1.13. Для систем типа атомов с помощью теоремы 1.5 можно получить простое достаточное условие того, что $\mu^{(\alpha)} \in \sigma_{\mathrm{d}}\left(H_{03}\left(\alpha, Z_{2}\right)\right)$ при $Z_{2} \in O(\alpha)$. Пусть, далее, система $Z_{1}=(1, \ldots, n)$ состоит из $(n-1)$ тождественных частищ с номерами $2,3, \ldots, n$ и зарядами $e_{i}=e, i=2, \ldots, n$, и частицы с номером 1 и зарядом $e_{1}=-(n-1) e, n \geqslant 2$. Пусть потенциалы взаимодействия

$$
V_{i j}\left(\left|r_{1}\right|\right)=e_{i} e_{j} \cdot\left|r_{1}\right|^{-\gamma}, \quad 0<\gamma<1.5 \text {. }
$$

Как показано в [10] без учета симметрии (с учетом симметрии это следует из [1]), множество $O(\alpha)$ содержит все двухкластерные распадения $Z_{2}(j)=\left\{C_{1 j}, C_{2 j}\right\}$, где $C_{1 j}=Z_{1} \backslash(j), C_{2 j}=j, j=2,3, \ldots, n$, и, значит, $\mu^{(\alpha)}=\inf H_{03}\left(\alpha ; Z_{2}(2)\right)$. Из теоремы 1.5 следует, что

$$
\mu\left(\alpha ; Z_{2}\right)=\inf H_{03}\left(\alpha ; Z_{3}(2,3)\right),
$$

где $Z_{3}(2,3)=\left\{Z_{1} \backslash(2,3) ;(2) ;(3)\right\}$. 
TеOPEMA 1.6. Пусmb

$$
\mu\left(\alpha ; Z_{2}\right) \in \sigma_{\mathrm{p}}\left(H_{03}\left(\alpha ; Z_{3}(2,3)\right)\right) .
$$

Тогда

$$
\mu^{(\alpha)} \in \sigma_{\mathrm{d}}\left(H_{03}\left(\alpha ; Z_{2}(2)\right)\right) .
$$

ЗАмечаниЕ. Так как операторы $H_{03}\left(\alpha ; Z_{2}(i)\right), i=2,3, \ldots, n$, и $H_{03}\left(\alpha ; Z_{3}(i, j)\right)$, $j \neq i, \quad i, j=2,3, \ldots, n$, унитарно эквивалентны соответственно $H_{03}\left(\alpha ; Z_{2}(2)\right)$ и $H_{03}\left(\alpha, Z_{3}(2,3)\right)$, то в формулировке теоремы 1.6 можно вместо $Z_{2}(2)$ и $Z_{3}(2,3)$ взять $Z_{2}(i)$ и $Z_{3}(i, j)$ при любых $i, j \geqslant 2, j \neq i$.

1.14. Доказательство теоремы 1.6 проводится в конце раздела 3 , а здесь мы очень просто покажем, что для трехчастичной системы $Z_{1}=\{1,2,3\}$ типа атома гелия условие (1.17) выполнено. (Напомним еше раз, что справедливость (1.17) для любых атомов установлена в [2].) В рассматриваемом случае $Z_{2}(2)=\{(1,3),(2)\}, Z_{3}=Z_{3}(2,3)=$ $\{(1),(2),(3)\}$ и оператор $H_{03}\left(Z_{3}\right)$ есть оператор энергии трех не взаимодействующих друг с другом частиц с отделенным движением каждой частицы в направлении оси $z$ и с фиксированным псевдомоментом. Другими словами, $H_{03}\left(Z_{3}\right)$ - это оператор, полученньй из оператора

$$
\mathcal{H}_{03}\left(Z_{3}\right)=\sum_{j=1}^{3} m_{j}^{-1}\left(\frac{1}{i} \nabla_{j \perp}-e_{j} A_{j}\right)^{2}
$$

после фиксации псевдомомента. Оператор $\mathcal{H}_{03}\left(Z_{3}\right)$ имеет чисто точечный спектр, накопляющийся к $+\infty$. Но каждая точка спектра оператора с фиксированным псевдомоментом есть точка спектра оператора энергии этой же системы до фиксации псевдомомента. Поэтому оператор $H_{03}\left(Z_{3}\right)$, как и $\mathcal{H}_{03}\left(Z_{3}\right)$, имеет чисто точечный спектр. Таким образом, (1.17) выполнено; значит, для трехчастичных систем типа атома гелия выполняется (1.18), а следовательно, и условие (1.7), т.е. теоремы 1.2, 1.4 применимы к $Z_{1}=\{1,2,3\}$

\section{2. ДОКАЗАТЕЛЬСТВО ТЕОРЕМЫ 1.2}

2.1. Основная идея доказательства состоит в построении таких подпространств функций $\mathcal{M}_{i}(\lambda), i=1,2$, что для всех $\lambda<0$

$$
\begin{array}{llll}
\left(H_{0}^{\alpha} \psi, \psi\right) \leqslant\left(\mu^{(\alpha)}+\lambda\right)\|\psi\|^{2}, & \text { если } & \psi \in \mathcal{M}_{1}(\lambda), \\
\left(H_{0}^{\alpha} \psi, \psi\right)>\left(\mu^{(\alpha)}+\lambda\right)\|\psi\|^{2}, & \text { если } & \psi \perp \mathcal{M}_{2}(\lambda),
\end{array}
$$

и в последующей оценке размерности этих подпространств через величины $N\left(\lambda ; h_{Z_{2}}^{ \pm}\right)$. Построение пространства $\mathcal{M}_{1}(\lambda)$, оценка его размерности и доказательство неравенства (2.1) делаются так же, как в [4]. Поэтому мы приводим здесь только определение $\mathcal{M}_{1}(\lambda)$. Построение пространства $\mathcal{M}_{2}(\lambda)$, оценка величины $\operatorname{dim} \mathcal{M}_{2}(\lambda)$ и доказательство неравенства (2.2) осушествляются с помошью одновременного применения подходов 
из [3] и [4]. При этом хотя $\mathcal{M}_{2}(\lambda)$ строится, как и в [4], на основе линейной оболочки произведений собственных функций одномерного эффективного оператора (отвечающих его собственным значениям, не превосходящим $\lambda$ ) и собственных функций основного состояния системы $Z_{2}$, определяющей границу сплошного спектра, но в отличие от [4] носители этих функций "срезаются" конусами относительного движения распавшейся системы не в направлении 3-й оси, а во всем пространстве, и лишь потом из этих конусов выделяются области, отвечаюшие распадениям только в направлении 3-й оси. Таким образом, мы проводим здесь двойное разбиение конфигурационного пространства $R_{0}$. Отметим, что разбиение пространства $R_{0}$ только в направлении оси $z$ может привести к цели лишь в случае $Q\left[C_{1}\right] Q\left[C_{2}\right]>0[4]$, который для нейтральных систем невозможен.

2.2. Приступаем к построению пространства $\mathcal{M}_{2}(\lambda)$ с требуемыми свойствами. Так как оператор $H_{0}^{(\alpha)}$ зависит только от относительных координат $q$, то мы можем воспользоваться рассуждениями работы [5], где магнитное поле отсутствует. А именно, по произвольному $\mathcal{E}>0$ строим такое конечномерное пространство $\mathcal{M}_{2}^{0}$, что при $\psi(q) \in \mathcal{D}\left(H_{0}^{(\alpha)}\right), \psi \perp \mathcal{M}_{2}^{0}$ выполняется неравенство

$$
\begin{aligned}
\left(H_{0}^{(\alpha)} \psi, \psi\right) \geqslant & \sum_{Z_{2} \in O(\alpha)}\left\{\left(H_{0}^{(\alpha)} \psi_{Z_{2}}, \psi_{Z_{2}}\right)-\mathcal{E}\left\|\psi_{Z_{2}}\left|\zeta_{Z_{2}}\right|^{-(\gamma+2) / 2}\right\|^{2}-\right. \\
& \left.-C(\mathcal{E})\left\|\psi_{Z_{2}} \chi\left(t_{Z_{2}}\right)\left|\zeta_{Z_{2}}\right|^{-1}\right\|^{2}\right\}+\mu^{(\alpha)}\left(\|\psi\|^{2}-\sum_{Z_{2} \in O(\alpha)}\left\|\psi_{Z_{2}}\right\|^{2}\right),
\end{aligned}
$$

где

$$
\begin{gathered}
\psi_{Z_{2}}=\psi u_{Z_{2}} \omega_{b}, \quad u_{Z_{2}}=u\left(t_{Z_{2}}\right), \quad \omega_{b}=\omega_{b}\left(\left|\zeta_{Z_{2}}\right|\right), \\
t_{Z_{2}}=\left|P_{0}\left(Z_{2}\right) q\right|_{1} \cdot\left|P_{c}\left(Z_{2}\right) q\right|_{1}^{-1},
\end{gathered}
$$

$P_{0}\left(Z_{2}\right)$ и $P_{c}\left(Z_{2}\right)$ - проекторы в смысле скалярного произведения $\left.(\cdot, \cdot)\right)_{1}($ см. $(1.3))$ соответственно на подпространства

$$
\begin{gathered}
R_{0}\left(Z_{2}\right)=\left\{q^{0}\left(Z_{2}\right)\right\}=\left\{q \mid q \in R_{0}, \sum_{j \in C_{t}} m_{j} q_{j}=0, t=1,2\right\} \\
R_{c}\left(Z_{2}\right)=\left\{q^{c}\left(Z_{2}\right)\right\}=\left\{q \mid q \in R_{0},\left(q, q^{0}\right)_{1}=0 \quad \forall q^{0} \in R_{0}\left(Z_{2}\right)\right\}, \\
\left|P_{0}\left(Z_{2}\right) q\right|_{1}^{2}=\frac{1}{2} \sum_{t=1}^{2} \frac{1}{M\left[C_{t}\right]} \sum_{i, j \in C_{t}} m_{i} m_{j}\left|q_{i}-q_{j}\right|^{2}, \\
\left|P_{c}\left(Z_{2}\right) q\right|_{1}^{2}=(q, q)-\left|P_{0}\left(Z_{2}\right) q\right|_{1}^{2}=M\left(Z_{2}\right)\left|\zeta_{Z_{2}}\right|^{2}
\end{gathered}
$$

(см. [11, с. 125]),

$$
\begin{gathered}
q_{i}-q_{j}=q_{i}^{0}\left(Z_{2}\right)-q_{j}^{0}\left(Z_{2}\right), \quad q_{j}^{0}\left(Z_{2}\right)=q_{j}-\zeta_{Z_{2}}\left[C_{t}\right], \quad j \in C_{t}, \\
\zeta_{Z_{2}}=\left(\zeta_{1}, \zeta_{2}, \zeta_{3}\right)=\left(\zeta_{\perp}, \zeta_{3}\right)=\zeta\left[C_{1}\right]-\zeta\left[C_{2}\right], \\
\zeta\left[C_{1}\right]=M\left[C_{t}\right]^{-1} \sum_{j \in C_{t}} m_{j} q_{j}, \quad t=1,2 ;
\end{gathered}
$$


$u(s), \omega_{b}(s) \in C^{2}[0,+\infty), u(s) \equiv 1$ при $0 \leqslant s \leqslant \delta, u(s) \equiv 0$ при $s \geqslant \beta>\delta, \omega_{b}(s) \equiv 0$ при $0 \leqslant s \leqslant b<b_{1}, \omega_{b}(s)=1$ при $s \geqslant b_{1}, \chi(q) \equiv 1$ при $q \in \Omega=\left\{q\left|t_{Z_{2}} \in[\delta, \beta],\right| \zeta_{Z_{2}} \mid \geqslant b\right\}$, $\chi(q) \equiv 0$ при $q \bar{\in} \Omega$.

В п. 2.3-2.7 мы оцениваем снизу правую часть неравенства (2.3). Используя полученную оценку, в п. 2.8 определяем подпространство $\widetilde{\mathcal{M}}_{2}(\lambda)$ так, что пространство $\mathcal{M}_{2}(\lambda):=\mathcal{M}_{2}^{0} \oplus \widetilde{\mathcal{M}}_{2}(\lambda)$ будет обладать требуемыми свойствами.

2.3. Обозначим через $\varphi_{i}\left(q_{Z_{2}}\right), \quad i=1,2, \ldots, i_{0}$, ортонормированные собственные функции, образующие канонический базис представления типа $\hat{\alpha}_{0}$ (см. п. 1.4) в собственном подпространстве $W\left(\alpha ; Z_{2}\right)$ оператора $H_{03}\left(\alpha ; Z_{2}\right)$, отвечаюшем собственному значению $\mu^{\alpha} ;$ здесь $i_{0}=\operatorname{dim} W\left(\alpha ; Z_{2}\right), q_{Z_{2}}=\left\{q_{1}\left(Z_{2}\right), \ldots, q_{n}\left(Z_{2}\right)\right\} \in R_{03}\left(Z_{2}\right), q_{i}\left(Z_{2}\right)=$ $\left\{q_{i 1}, q_{i 2}, q_{i 3}^{0}\left(Z_{2}\right)\right\}$. В дальнейших оценках мы неоднократно будем использовать два следуюших свойства функций $\varphi_{i}\left(q_{Z_{2}}\right)$ :

$$
\varphi\left(q_{Z_{2}}\right)\left|q_{Z_{2}}\right|^{k} \in \mathcal{L}_{2}\left(R_{03}\left(Z_{2}\right)\right), \quad k=1,2,
$$

и

$$
\left(L \varphi_{i}, \varphi_{j}\right)_{\mathcal{L}_{2}\left(R_{03}\left(Z_{2}\right)\right)}=0, \quad i \neq j
$$

для любой функшии $L=L\left(q_{Z_{2}}, \zeta_{Z_{2}}\right)$, инвариантной относительно перестановок группы $\widehat{S}\left(Z_{2}\right)$ и такой, что $L \varphi_{j} \in \mathcal{L}_{2}\left(R_{03}\left(Z_{2}\right)\right)$ при любом фиксированном $\zeta_{Z_{2}}$. Соотношение (2.4) доказывается аналогично лемме 3.1 работы [4]. Равенство (2.5) является следствием того факта, что функции $L \varphi_{i}$ и $\varphi_{j}$ принадлежат разньм строкам неприводимого представления типа $\hat{\alpha}_{0}$ : этим свойством обладают функции $\varphi_{i}, \varphi_{j}$ при $i \neq j$ и оно сохраняется при умножении $\varphi_{i}$ на функцию $L\left(q_{Z_{2}} \zeta_{Z_{2}}\right)$, инвариантную относительно перестановок из $S\left(Z_{2}\right)$.

Далее мы будем использовать разложение функции $\psi_{Z_{2}}$ по функциям $\varphi_{i}\left(q_{Z_{2}}\right)$ :

$$
\psi_{Z_{2}}(q)=\sum_{i=1}^{i_{0}} \varphi_{i}\left(q_{Z_{2}}\right) \Phi_{i}\left(\zeta_{3}\right)+g(q)
$$

где $\zeta_{3}=\zeta_{3}\left(Z_{2}\right), \Phi_{i}\left(\zeta_{3}\right)=\left(\psi_{Z_{2}}, \varphi_{i}\right)_{\mathcal{L}_{2}\left(R_{03}\left(Z_{2}\right)\right)}$ и, очевидно,

$$
\left(g(q), \varphi_{i}\left(q_{Z_{2}}\right)\right)_{\mathcal{L}_{2}\left(R_{03}\left(Z_{2}\right)\right)}=0 \quad \forall \zeta_{3} .
$$

Пусть $\chi_{1}(q), \chi_{2}(q)$ и $\chi_{0}(q)$ - характеристические функции областей

$$
\begin{aligned}
& \Omega_{1}=\Omega_{1}(\beta)=\left\{q \mid q \in R_{0}, t_{Z_{2}} \leqslant \beta\right\}, \\
& \Omega_{2}=\Omega_{2}(b)=\left\{q\left|q \in R_{0},\right| \zeta_{Z_{2}} \mid \geqslant b\right\}, \\
& \Omega_{0}=\Omega_{0}(\beta, b)=\Omega_{1} \cap \Omega_{2} .
\end{aligned}
$$

Очевидно, $\chi_{0}=\chi_{1} \chi_{2}$. В силу $(2.6)$

$$
\psi_{Z_{2}}(q)=\sum_{i=1}^{i_{0}} \varphi_{i}\left(q_{Z_{2}}\right) \Phi_{i}\left(\zeta_{3}\right) \chi_{j}+g(q) \chi_{j}, \quad j=0,2 .
$$


Начинаем последовательно оценивать величины в правой части неравенства (2.3). Фиксируем $Z_{2}$ и всюду далее для краткости пишем $\zeta$ вместо $\zeta_{Z_{2}}$. Очевидно,

$$
|\zeta|^{-1} \chi_{j}(q) \leqslant B_{b}\left(\zeta_{3}\right), \quad j=0,2,
$$

где $B_{b}\left(\zeta_{3}\right)=\left|\zeta_{3}\right|^{-1}$ при $\left|\zeta_{3}\right| \geqslant b, B_{b}\left(\zeta_{3}\right)=b^{-1}$ при $\left|\zeta_{3}\right| \leqslant b$. Поэтому и в силу $(2.5),(2.8)$ при больших $b=b(\mathcal{E})$

$$
\begin{aligned}
\left\|\psi_{Z_{2}}|\zeta|^{-(\gamma+2) / 2}\right\|^{2} & \leqslant \mathcal{E}\|g\|^{2}+\sum_{i, j=1}^{i_{0}}\left(B_{b}^{\gamma+2}\left(\zeta_{3}\right) \varphi_{i} \Phi_{i}, \varphi_{j} \Phi_{j}\right) \leqslant \\
& \leqslant \mathcal{E}\|g\|^{2}+\sum_{i=1}^{i_{0}}\left\|B_{b}^{\frac{\gamma+2}{2}}\left(\zeta_{3}\right) \Phi_{i}\left(\zeta_{3}\right)\right\|^{2} .
\end{aligned}
$$

Так как $q_{\left(Z_{2}\right)}^{0}=P_{0}\left(Z_{2}\right) q=\left\{q_{1}^{0}\left(Z_{2}\right), \ldots, q_{n}^{0}\left(Z_{2}\right)\right\}$, где $q_{i}^{0}\left(Z_{2}\right)=q_{i}-\zeta\left[C_{t}\right]$ при $i \in C_{t}$, то

$$
\left|P_{0}\left(Z_{2}\right) q\right|_{1} \leqslant\left|q_{Z_{2}}\right|_{1}
$$

и при $q \in \operatorname{supp} u_{Z_{2}}$

$$
\frac{\left|q_{\left(Z_{2}\right)}\right|}{\delta \cdot M\left(Z_{2}\right) \cdot|\zeta|} \geqslant \frac{\left|P_{0}\left(Z_{2}\right) q\right|_{1}}{\delta\left|P_{c}\left(Z_{2}\right) q\right|_{1}} \geqslant 1 .
$$

При $b>b(a)$ в силу $(2.8),(2.9),(2.11)$ и $(2.4)($ c $k=1)$

$$
\begin{aligned}
\left\|\psi_{Z_{2}} \chi \chi_{2}|\zeta|^{-1}\right\|^{2} & \leqslant 2\left\|\sum_{i=1}^{i_{0}} \varphi_{i} \cdot \Phi_{i} \cdot B_{b} \chi \chi_{2}\right\|^{2}+2\left\|g B_{b}\right\|^{2} \leqslant \\
& \leqslant c \sum_{i=1}^{i_{0}}\left\|\Phi_{i}\left(\zeta_{3}\right) B_{b}^{3 / 2}\left(\zeta_{3}\right)\right\|^{2}+\mathcal{E}\|g\|^{2} .
\end{aligned}
$$

Здесь и далее через $c$ мы будем обозначать константы (возможно, зависящие от $\mathcal{E}$ ), величина которых не играет роли для доказательства.

2.4. Переходим к оценкам главного члена в неравенстве (2.3) - квадратичной формы $\left(H_{0}^{(\alpha)} \psi_{Z_{2}}, \psi_{Z_{2}}\right)$. Очевидно,

$$
\left(H_{0} \psi_{Z_{2}}, \psi_{Z_{2}}\right)=\left(\left(H_{03}\left(Z_{2}\right)+I_{Z_{2}}-\frac{1}{M\left(Z_{2}\right)} \frac{d^{2}}{d \zeta_{3}^{2}}\right) \psi_{Z_{2}}, \psi_{Z_{2}}\right),
$$

где $I_{Z_{2}}=V(q)-V_{Z_{2}}(q)$. В силу $(2.6)$

$$
\left(H_{03}\left(Z_{2}\right) \psi_{Z_{2}}, \psi_{Z_{2}}\right) \geqslant \mu^{(\alpha)}\left\|\psi_{Z_{2}}\right\|^{2}+\delta_{0}\|g\|^{2},
$$

где $\delta_{0}>0$ - расстояние от $\mu^{(\alpha)}$ до ближайшей точки спектра оператора $H_{0}\left(\alpha ; Z_{2}\right)$. Далее, очевидно,

$$
-\frac{1}{M\left(Z_{2}\right)}\left(\frac{d^{2}}{d \zeta_{3}^{2}} \psi_{Z_{2}}, \psi_{Z_{2}}\right) \geqslant-\frac{1}{M\left(Z_{2}\right)} \sum_{i=1}^{i_{0}}\left(\frac{d^{2}}{d \zeta_{3}^{2}} \Phi_{i}, \Phi_{i}\right) .
$$


Основные трудности в оценке величины $\left(H_{0}^{(\alpha)} \psi_{Z_{2}}, \psi_{Z_{2}}\right)$ связаны с оценкой формы $\left(I_{Z_{2}} \psi_{Z_{2}}, \psi_{Z_{2}}\right)$. Используя $(2.8)$ с $j=0$, имеем

$$
\begin{aligned}
\left(I_{Z_{2}} \psi_{Z_{2}}, \psi_{Z_{2}}\right)= & \left(\chi_{0} I_{Z_{2}} g, g\right)+\left(\chi_{0} I_{Z_{2}} \sum_{i=1}^{i_{0}} \varphi_{i} \Phi_{i}, \sum_{t=1}^{i_{0}} \varphi_{t} \Phi_{t}\right)+ \\
& +2 \operatorname{Re}\left(\chi_{0} I_{Z_{2}} \sum_{i=1}^{i_{0}} \varphi_{i} \Phi_{i}, g\right) .
\end{aligned}
$$

2.5. Пусть $i \in C_{1}, j \in C_{2}$. Тогда

$$
\left|q_{i}-q_{j}\right|=\left|q_{i}-\zeta\left[C_{1}\right]+\zeta\left[C_{2}\right]-q_{j}+\zeta Z_{2}\right|=\left|q_{i}^{0}\left(Z_{2}\right)-q_{j}^{0}\left(Z_{2}\right)+\zeta\right| .
$$

Из п. 2.2 следует, что при $q \in \Omega_{1}(\beta)$

$$
\left|q_{t}^{0}\left(Z_{2}\right)\right| \leqslant c \beta|\zeta|
$$

и поэтому при малом $\beta$ и $\forall q \in \Omega_{1}(\beta)$

$$
\left|q_{i}-q_{j}\right| \geqslant \frac{|\zeta|}{2} .
$$

При $q \in \Omega_{0}$ выполняется $|\zeta| \geqslant b$. Поэтому и в силу $(2.17)$ при $b \geqslant 2 a$ (см. п. 1.1)

$$
\chi_{0} V_{i j}\left(\left|q_{i}-q_{j}\right|\right)=e_{i} e_{j}\left|q_{i}-q_{j}\right|^{-\gamma} \cdot \chi_{0} .
$$

Следовательно, при большом $b$ и малом $\beta$

$$
\left|\left(\chi_{0} I_{Z_{2}} g, g\right)\right| \leqslant \mathcal{E}\|g\|^{2} .
$$

Для оценки других членов в $(2.16)$ величину $\left|q_{i}-q_{j}\right|^{-\gamma}=\left|q_{i}^{0}\left(Z_{2}\right)-q_{j}^{0}\left(Z_{2}\right)+\zeta\right|^{-\gamma}$ при $q \in$ $\Omega_{0}(\beta, b), \beta \ll 1$ разложим сначала по степеням $|\zeta|^{-1}$, а потом главньй член полученного разложения - по степеням $\left|\zeta_{3}\right|^{-1}$. Имеем

$$
\chi_{0}\left|q_{1}^{0}\left(Z_{2}\right)-q_{j}^{0}\left(Z_{2}\right)+\zeta\right|^{-\gamma}=\chi_{0}|\zeta|^{-\gamma}+\gamma\left(\zeta_{3}, q_{i 3}^{0}-q_{j 3}^{0}\right)|\zeta|^{-\gamma-2} \chi_{0}+F_{1},
$$

где с учетом $(2.9),(2.11)$

$$
\left|F_{1}\right| \leqslant c \chi_{0}\left(\left|q_{i}^{0}\left(Z_{2}\right)\right|^{2}+\left|q_{j}^{0}\left(Z_{2}\right)\right|^{2}\right)|\zeta|^{-\gamma-2} \leqslant c\left|q\left(Z_{2}\right)\right|^{2} B_{b}^{\gamma+2}\left(\zeta_{3}\right) .
$$

Далее, пусть $\Omega_{3}(\beta)=\left\{q\left|q \in \Omega_{0},\right| \zeta_{\perp}\left|\leqslant \beta_{1}\right| \zeta_{3} \mid\right\}, \Omega_{4}=\Omega_{0}-\Omega_{3}$ и $\chi_{j}$ - характеристическая функция области $\Omega_{j}(\beta), j=3,4$. Тогда для любого $p>0$

$$
\chi_{0}|\zeta|^{-p}=\chi_{0}\left(\chi_{3}+\chi_{4}\right)|\zeta|^{-p}=\chi_{0} \chi_{3}\left|\zeta_{3}\right|^{-p}+\chi_{0} \chi_{4}|\zeta|^{-p}+F_{2},
$$

где в силу $(2.9),(2.11)$

$$
\begin{gathered}
\chi_{0} \chi_{4}|\zeta|^{-p} \leqslant \chi_{4} B_{b}^{p}\left(\zeta_{3}\right) \leqslant B_{b}^{p+2}\left(\zeta_{3}\right) b^{2}\left|\zeta_{\perp}\right|^{2} \beta_{1}^{-2}, \\
\left|F_{2}\right| \leqslant \frac{p}{2} \chi_{0} \chi_{3}\left|\zeta_{\perp}\right|^{2} B_{b}^{p+2}\left(\zeta_{3}\right) .
\end{gathered}
$$

Подставим в правую часть (2.21) полученное в (2.22) выражение для $\chi_{0}|\zeta|^{-p}$ с $p=\gamma$, умножим полученное равенство на $e_{i} e_{j}$ и просуммируем по всем $i \in C_{1}, j \in C_{2}$. Тогда получим

$$
\begin{aligned}
\chi_{0} I_{Z_{2}} & =Q\left|\zeta_{3}\right|^{-\gamma} \chi_{0} \chi_{3}+|\zeta|^{-\gamma-2} \sum_{i \in C_{1}, j \in C_{2}} e_{i} e_{j} \zeta_{3}\left(q_{i 3}^{0}\left(Z_{2}\right)-q_{j 3}^{0}\left(Z_{2}\right)\right) \chi_{0}+F_{3}, \\
\text { где }\left|F_{3}\right| & \leqslant c B_{b}^{\gamma+2}\left(\zeta_{3}\right)\left|q\left(Z_{2}\right)\right|^{2} .
\end{aligned}
$$


2.6. Возврашаемся к оценке членов в (2.16). В силу (2.5) с $L=\chi_{0} I_{Z_{2}}$ имеем

$$
\left(\chi_{0} I_{Z_{2}} \sum_{i=1}^{i_{0}} \varphi_{i} \Phi_{i}, \sum_{j=1}^{i_{0}} \varphi_{j} \Phi_{j}\right)=\sum_{j=1}^{i_{0}}\left(\chi_{0} I_{Z_{2}} \varphi_{j} \Phi_{j}, \varphi_{j} \Phi_{j}\right) .
$$

Далее, вследствие условия $A_{2}$ (п. 1.5) и т.к. гамильтониан $H_{03}\left(Z_{2}\right)$ инвариантен относительно инверсии $q_{j 3}^{0} \leftrightarrow-q_{j 3}^{0}$ (но не $\left.q_{j}^{0} \leftrightarrow-q_{j}^{0} !\right)$, функции $\varphi_{j}$ имеют определенную четность и функции $\left|\varphi_{j}\right|^{2}$ являются четными относительно замены $q_{j 3}^{0} \rightarrow-q_{j 3}^{0}$. Поэтомy

$$
\int \chi_{0} \zeta_{3}|\zeta|^{-\gamma-2}\left(q_{i 3}^{0}\left(Z_{2}\right)-q_{j 3}^{0}\left(Z_{2}\right)\right)\left|\varphi_{t}\left(q_{Z_{2}}\right)\right|^{2} d R_{03}\left(Z_{2}\right)=0 .
$$

Следовательно, в силу (2.23)

$$
\left(I_{Z_{2}} \chi_{0} \varphi_{j} \Phi_{j}, \varphi_{j} \Phi_{j}\right)=Q\left(Z_{2}\right) \int\left|\zeta_{3}\right|^{-\gamma} \chi_{0} \chi_{3}\left|\varphi_{j} \Phi_{j}\right|^{2} d R_{03}\left(Z_{2}\right)+\left(F_{3} \varphi_{j} \Phi_{j}, \varphi_{j} \Phi_{j}\right) .
$$

Так как исходная система была нейтральна, подсистемы $C_{1}, C_{2}$ из $Z_{2}$ или обе нейтральны $\left(Q\left(Z_{2}\right)=0\right)$, или заряжены разноименно $\left(Q\left(Z_{2}\right)<0\right)$. Поэтому и в силу $(2.4)$

$$
\begin{aligned}
\left(I_{Z_{2}} \chi_{1} \varphi_{j} \Phi_{j}, \varphi_{j} \Phi_{j}\right) \geqslant & Q\left(Z_{2}\right) \int_{\left|\zeta_{3}\right| \geqslant b_{0}}\left|\Phi_{j}\left(\zeta_{3}\right)\right|^{2}\left|\zeta_{3}\right|^{-\gamma} d \zeta_{3}- \\
& -c \int\left|\Phi_{j}\left(\zeta_{3}\right)\right|^{2} B_{b}^{\gamma+2}\left(\zeta_{3}\right) d \zeta_{3},
\end{aligned}
$$

где $c$ - некоторая константа, $b_{0}=\left(1+\beta_{1}^{2}\right)^{-1 / 2} b$. Здесь и далее мы используем тот факт, что при $\chi_{0} \chi_{3}=1$ вьполняются неравенства $b^{2} \leqslant \zeta_{\perp}^{2}+\zeta_{3}^{2} \leqslant \zeta_{3}^{2}\left(1+\beta_{1}^{2}\right)$, т.е. $\left|\zeta_{3}\right| \geqslant b_{0}$.

2.7. Оценим, наконец, перекрестные члены в (2.15). Пусть $\chi_{5}\left(\zeta_{3}\right)=0$ при $\left|\zeta_{3}\right|<b_{0}$, $\chi_{5}\left(\zeta_{3}\right)=1$ при $\left|\zeta_{3}\right| \geqslant b_{0}$. Тогда $\chi_{0} \chi_{3} \chi_{5}=\chi_{0} \chi_{3}$ и в силу $(2.23)$

$$
\left(\chi_{0} I_{Z_{2}} \varphi_{j} f_{j}, g\right)=Q\left(\left|\zeta_{3}\right|^{-\gamma} \chi_{5}\left(\zeta_{3}\right) \varphi_{j} \Phi_{j}, g\right)+\left(F_{4} \varphi_{j} \Phi_{j}, g\right)+\left(F_{5} \varphi_{j} \Phi_{j}, g\right),
$$

где

$$
F_{4}=Q\left(\chi_{1} \chi_{3}-1\right) \chi_{5}\left|\zeta_{3}\right|^{-\gamma}, \quad\left|F_{5}\right| \leqslant c\left|q^{0}\left(Z_{2}\right)\right|^{2} B_{b}^{\gamma+1} .
$$

В силу $(2.7)$

$$
\left(\left|\zeta_{3}\right|^{-\gamma} \chi_{5}\left(\zeta_{3}\right) \varphi_{j} \Phi_{j}, g\right)=0 .
$$

Второе и третье слагаемые в (2.26) оцениваются по неравенству Буняковского:

$$
\left|\left(F_{t} \varphi_{j} \Phi_{j}, g\right)\right| \leqslant \mathcal{E}\|g\|^{2}+c\left\|F_{t} \varphi_{j} \Phi_{j}\right\|^{2}, \quad t=4,5 .
$$

В силу (2.4)

$$
\left\|F_{5} \varphi_{j} \Phi_{j}\right\|^{2} \leqslant c\left\|B_{b}^{\gamma+1}\left(\zeta_{3}\right) \Phi_{j}\right\|^{2}
$$


Ясно, что $F_{4} \neq 0$, только если $\chi_{0}=0, \chi_{5}=1$ или $\chi_{3}=0, \chi_{5}=1$. Если $\left|\zeta_{3}\right| \geqslant b$ (и, значит, $|\zeta| \geqslant b$ ), то равенства $\chi_{0}=0, \chi_{5}=1$ означают, что $\chi_{1}=0$ (см. п. 2.3), т.е. что

$$
\left|P_{0}\left(Z_{2}\right) q\right|_{1} \geqslant \beta\left|P_{c}\left(Z_{2}\right) q\right|_{1}=\beta M\left(Z_{2}\right)\left|\zeta_{3}\right|
$$

а равенства $\chi_{3}=0, \chi_{5}=1$ эквивалентны условию

$$
\left|\zeta_{\perp}\right| \geqslant \beta_{1}\left|\zeta_{3}\right| \geqslant \beta_{1} b
$$

Если $b_{0} \leqslant\left|\zeta_{3}\right|<b$ (при $\left|\zeta_{3}\right|<b_{0}$ выполняется $F_{4} \equiv 0$ ), то, очевидно, $b \cdot\left|\zeta_{3}\right|^{-1} \geqslant \beta_{1}$. В силу сказанного при $q \in \operatorname{supp} F_{4}$

$$
\tau:=\left(\left|P_{0}\left(Z_{2}\right) q\right|_{1}+\left|\zeta_{\perp}\right|+b\right)\left|\zeta_{3}\right|^{-1} \geqslant \beta_{2}:=\min \left\{\beta_{1}, 1, \beta M\left(Z_{2}\right)\right\}
$$

и в силу (2.4)

$$
\left\|F_{4} \varphi_{j} \Phi_{j}\right\|^{2} \leqslant\left\|F_{4} \tau \beta_{2}^{-1} \varphi_{j} \Phi_{j}\right\|^{2} \leqslant c\left\|\chi_{5}\left|\zeta_{3}\right|^{-\gamma-1} \Phi_{j}\right\|^{2} .
$$

Из соотношений (2.26)-(2.30) вытекает, что

$$
\left|\left(\chi_{0} I_{Z_{2}} \varphi_{j} \Phi_{j}, g\right)\right| \leqslant \mathcal{E}\|g\|^{2}+c\left\|B_{b}^{\gamma+1}\left(\zeta_{3}\right) \Phi_{j}\right\|^{2}
$$

2.8. Используя $(2.3),(2.10),(2.12)-(2.14),(2.16),(2.20)$ и (2.31), мы получаем, что при $\psi \perp \mathcal{M}_{2}^{0}(\lambda)$ (см. п. 2.2)

$$
\left(H_{0}^{\alpha} \psi, \psi\right) \geqslant \mu^{\alpha}\|\psi\|^{2}+\sum_{Z_{2} \in O(\alpha)} \sum_{j=1}^{i_{0}}\left(h_{Z_{2}}^{+} \Phi_{j}, \Phi_{j}\right)
$$

где аргументы $\zeta_{3}$ функций $\Phi_{j}\left(\zeta_{3}\right)$ зависят от $Z_{2}$, но мы не указываем на эту зависимость, чтобы не усложнять обозначения ${ }^{5)}$. Неравенство (2.32) подсказьвает, как определить пространство $\widetilde{\mathcal{M}}_{2}(\lambda)$, чтобы при $\psi \perp\left(\widetilde{\mathcal{M}}_{2}(\lambda) \oplus \mathcal{M}_{2}^{0}\right)$ выполнялось неравенство (2.2). А именно, положим

$$
\widetilde{\mathcal{M}}_{2}(\lambda)=\left\{\tilde{\psi} \mid \tilde{\psi}=P^{(\alpha)} \sum_{g \in S} \sum_{i, j} c_{i j}(g) T_{g} \varphi_{i}\left(q_{Z_{2}}\right) f_{j}\left(\zeta_{3}\right) u_{Z_{2}} \omega_{b}\right\},
$$

где $f_{j}\left(\zeta_{3}\right)$ - собственные функции оператора $h_{Z_{2}}^{+}$, отвечающие его собственным значениям, не превосходяшим $\lambda$, функции $u_{Z_{2}}=u\left(t_{Z_{2}}\right), \omega_{b}=\omega_{b}\left(\zeta_{Z_{2}}\right), \varphi_{i}\left(q_{Z_{2}}\right)-$ те же, что в п. $2.2, c_{i j}(g)$ - произвольные числа. Пусть

$$
\mathcal{M}_{2}(\lambda)=\mathcal{M}_{2}^{0}+\widetilde{\mathcal{M}}_{2}(\lambda) .
$$

Тогда при $\psi \perp \mathcal{M}_{2}(\lambda)$ разложения (2.6) функций $\psi_{Z_{2}} \equiv \psi u_{Z_{2}} \omega_{b}$ при любом $Z_{2} \in O(\alpha)$ не могут содержать слагаемых $\varphi_{j}\left(q_{Z_{2}}\right) \Phi_{j}\left(\zeta_{3}\right)$ с такими функциями $\Phi_{j}\left(\zeta_{3}\right)$, для которых

$$
\left(h_{Z_{2}}^{+} \Phi_{j}, \Phi_{j}\right)<\lambda\left\|\Phi_{j}\right\|^{2}
$$

\footnotetext{
${ }^{5)}$ Если не выполнено условие $A_{1}$, то вид функции $\Phi_{j}$ также зависит от $Z_{2}$.
} 
Поэтому и так как

$$
\left\|\psi_{Z_{2}}\right\|^{2} \geqslant \sum_{j=1}^{i_{0}}\left\|\Phi_{j}\right\|^{2}, \quad\|\psi\|^{2} \geqslant \sum_{Z_{2} \in O(\alpha)}\left\|\psi_{Z_{2}}\right\|^{2},
$$

мы получаем, что

$$
\sum_{Z_{2} \in O(\alpha)} \sum_{j=1}^{i_{0}}\left(h_{Z_{2}}^{+} \Phi_{j}, \Phi_{j}\right) \geqslant \lambda\|\psi\|^{2} .
$$

Отсюда и из (2.32) следует неравенство (2.2).

Оценка

$$
\operatorname{dim} \mathcal{M}_{2}(\lambda) \leqslant d_{Z_{2}}\left(\alpha ; \tilde{\alpha}_{0}\right) N\left(\lambda ; h_{Z_{2}}^{+}\right)+\text {const }
$$

вытекает из леммы 4.2 работы [4].

2.9. Для завершения доказательства теоремы 1.2 строим пространство $\mathcal{M}_{1}(\lambda)$ так, чтобы при $\psi \in \mathcal{M}_{1}(\lambda)$ выполнялось неравенство (2.1) и чтобы

$$
\operatorname{dim} \mathcal{M}_{1}(\lambda) \geqslant d_{Z_{2}}\left(\alpha ; \tilde{\alpha}_{0}\right) N\left(\lambda ; h_{Z_{2}}^{-}\right)-c .
$$

Пусть

$$
\mathcal{M}_{1}(\lambda)=\left\{\psi \mid \psi \in P^{(\alpha)} \sum_{i, j} \sum_{g \in S} c_{i j}(g) T_{g}\left(\varphi_{i}\left(q_{Z_{2}}\right) f_{j}\left(b_{0}, \zeta_{3}\left(Z_{2}\right)\right) u\left(\tau_{3}\left(Z_{2}\right)\right)\right)\right\},
$$

где $f_{j}\left(b_{0}, \zeta_{3}\left(Z_{2}\right)\right)$ - собственные функции эффективного оператора $h_{Z_{2}}^{-}$в области $\left|\zeta_{3}\left(Z_{2}\right)\right| \geqslant b_{0}$ с нулевым граничным условием при $\left|\zeta_{3}\left(Z_{2}\right)\right|=b_{0}$, отвечающие всем его собственным значениям, меншшим $\lambda ; \varphi_{i}\left(q_{Z_{2}}\right), \quad i=1,2, \ldots, i_{0}$, - те же, что в п. $2.3 ; u(s)$ - та же, что в п. $2.2 ; \tau_{3}\left(Z_{2}\right)=\left|P_{03}\left(Z_{2}\right) q^{(3)}\right|_{1} \cdot\left|\zeta_{3}\left(Z_{2}\right)\right|^{-1}, \quad q^{(3)}=$ $\left(q_{1}^{(3)}, \ldots, q_{n}^{(3)}\right) \in R_{0}, \quad q_{j}^{(3)}=\left(0,0, q_{j 3}\right), \quad j=1, \ldots, n ; \quad c_{i j}(g)$ - произвольные числа. Пространство (2.34) с точностью до обозначений совпадает с пространством $\mathcal{M}_{1}(\lambda)$ из [4] (формула (2.5)), и проверка свойств $(2.33)$ и (2.1) проводится в точности так же, как в [4]. Теорема 1.2 доказана.

\section{3. ДОКАЗАТЕЛЬСТВА ТЕОРЕМ 1.5, 1.6}

3.1. Доказательство теоремы 1.5 (п. 3.2-3.8) по внешней схеме напоминает доказательство теоремы 2.1 работы [12]. Как и там, для оценки квадратичной формы рассматриваемого оператора по последовательностям $g_{k}$, описываюшим уход системы из любой ограниченной области конфигурационного пространства, мы делаем сначала разбиение этого пространства в направлении оси $z$, а потом - в плоскости $(x, y)$. Однако фактическое наполнение этой схемы иное. В направлении оси $z$ мы проводим сначала разбиение в конфигурационном пространстве кластера $C_{1}$ (независимо от состояния $C_{2}$ ), а потом разбиение в конфигурационном пространстве кластера $C_{2}$, когда кластер $C_{1}$ находится

2 Теоретическая и математическая физика, т. 118, № 1, 1999 г. 
в ограниченной области. В плоскости $(x, y)$ разбиение стандартное, однако с его помощью впервые устанавливается, что в области, отвечаюшей уходу кластеров $C_{1}, C_{2}$ друг от друга в плоскости $(x, y)$ (без их распадения), норма волновых функций $g_{k}$ стремится к нулю, т.е. что такой уход невозможен.

Теорема 1.6 доказывается стандартным образом, но с решаюшим использованием теоремы 1.5.

3.2. Пусть распадение $Z_{2}=\left\{C_{1}, C_{2}\right\}$ фиксировано и $g_{k}(q)$ - любая последовательность из $P^{(\alpha)} C_{0}^{2}\left(R_{03}\left(Z_{2}\right)\right)$ такая, что

$$
\left\|g_{k}\right\|=1, \quad \sup _{k}\left(H_{03}\left(Z_{2}\right) g_{k}, g_{k}\right)<+\infty \quad \text { и } \quad g_{k}(q) \rightarrow 0 \quad \text { в } \quad \mathcal{L}_{2}\left(R_{03}\left(Z_{2}\right)\right) .
$$

Мы докажем, что

$$
\underline{\lim }\left(H_{03}\left(\alpha ; Z_{2}\right) g_{k}, g_{k}\right) \geqslant \mu\left(\alpha ; Z_{2}\right)
$$

где

$$
\mu\left(\alpha ; Z_{2}\right)=\min _{Z_{s}^{\prime}, Z_{s}^{\prime}<Z_{2}, s \geqslant 3} \inf H_{03}\left(\alpha, Z_{s}\right) .
$$

Отсюда будет следовать, что

$$
\sigma_{\mathrm{ess}}\left(H_{03}\left(\alpha ; Z_{2}\right)\right) \subseteq\left[\mu\left(\alpha, Z_{2}\right),+\infty\right) .
$$

Доказательство противоположного включения проводится стандартным методом по образцу $[13$, п. $2.7,2.8]$ и здесь опускается.

3.3. Введем необходимые обозначения. Пусть $Y_{s j}=\left\{C_{1 j}, \ldots, C_{s j}\right\}-$ произвольное разбиение кластера $C_{j}$ на $s$ не взаимодействуюших между собой кластеров $C_{p j}$, $1 \leqslant p \leqslant s \leqslant n_{j}, \bigcup_{p} C_{p j}=C_{j}, C_{p j} \cap C_{l j}=\varnothing, p \neq l$. Введем в рассмотрение пространства

$$
\begin{aligned}
R_{03}\left[Y_{s j}\right]= & \left\{q \mid q=\left(q_{1} \ldots q_{n}\right), q_{i}=(0,0,0), i \bar{\in} C_{j} ; q_{i}=\left(0,0, q_{i 3}\right), i \in C_{j},\right. \\
& \left.\sum_{t \in C_{p j}} m_{t} q_{t 3}=0, p=1,2, \ldots, s\right\}, \\
R_{c 3}\left[Y_{s j}\right]= & \left\{q \mid q=\left(q_{1} \ldots q_{n}\right), q_{i}=(0,0,0), i \bar{\in} C_{j} ; q_{i}=\left(0,0, q_{i 3}\right), i \in C_{j},\right. \\
& \left.\left(q, q^{\prime}\right)_{1}=0 \quad \forall q^{\prime} \in R_{03}\left[Y_{s j}\right]\right\}
\end{aligned}
$$

и проекторы (в смысле $\left.(\cdot, \cdot))_{1}\right) P_{\kappa 3}\left[Y_{s j}\right]$ в пространстве $R_{03}\left(Z_{2}\right)$ на $R_{\kappa 3}\left[Y_{s j}\right], \kappa=0, c$. Положим

$$
\tau\left(Y_{1 j}\right)=\sum_{p \in C_{j}} m_{p} q_{p 3}^{2}, \quad \tau\left(Y_{s j}\right)=\left|P_{03}\left(Y_{s j}\right) q\right|_{1} \cdot\left|P_{c 3}\left(Y_{s j}\right) q\right|_{1}^{-1} .
$$

Выберем, далее, числа $b_{1}>a_{1} \gg 1$ и $a_{j}<b_{j} \ll 1,2 \leqslant j \leqslant n_{i}=\left|C_{i}\right|$, так же, как в п. 2.4 работы [13], но не для всей системы, а для ее подсистемы $C_{i}$ и определим вещественные дважды непрерывно дифференцируемые функции $u_{s j}(\tau), v_{s j}(\tau)$ так, что $0 \leqslant u_{s j}(\tau)$, $v_{s j}(\tau) \leqslant 1, u_{s j}^{2}(\tau)+v_{s j}^{2}(\tau)=1, u_{s j}(\tau)=1$ при $0 \leqslant \tau \leqslant a_{j}, u_{s j}(\tau)=0$ при $\tau \geqslant b_{j}$. Пусть

$$
u_{Y_{s j}}=u_{s j}\left(\tau\left(Y_{s j}\right)\right), \quad v_{Y_{s j}}=v_{s j}\left(\tau\left(Y_{s j}\right)\right) \text {. }
$$


3.4. Проведем оценку квадратичной формы $\left(H_{03}\left(\alpha ; Z_{2}\right) g_{k}, g_{k}\right)$ (см. п. 3.2), разбивая пространство относительного движения кластера $C_{1}$ в направлении 3 -й оси с помощью введенных функций $u_{Y_{s 1}}, v_{Y_{s 1}}$. Пусть

$$
\begin{aligned}
& \psi(q)=g_{k}(q), \quad \hat{\psi}_{0}=\psi, \quad \hat{\psi}_{j}=\hat{\psi}_{j-1} \cdot\left(1-\sum_{Y_{j, 1}} u_{Y_{j, 1}}^{2}\right)^{1 / 2} \\
& \psi_{j-1, Y_{j 1}}=\hat{\psi}_{j-1} u_{Y_{j 1}}, \quad j=1,2, \ldots, n_{1} \text {; } \\
& \psi_{j}=\sum_{Y_{j 1}} \psi_{j-1, Y_{j 1}}, \quad j=1,2, \ldots, n-1 ; \quad Z_{s 1}=\left\{Y_{s 1}, C_{2}\right\}, \quad Z_{s 2}=\left\{C_{1}, Y_{s 2}\right\}
\end{aligned}
$$

Аналогично соотношениям $(2.5),(2.6)$ из [13] мы получаем, что для произвольного $\mathcal{E}>0$ и $a_{1}=a_{1}(\mathcal{E}), b_{1}=b_{1}(\mathcal{E})$

$$
\left(H_{03}\left(Z_{2}\right) g_{k}, g_{k}\right) \geqslant \sum_{j=0}^{n_{1}-1} \sum_{Z_{j+1,1}}\left(H_{03}\left(Z_{j+1,1}\right) \psi_{j, Y_{j+1,1}}, \psi_{j, Y_{j+1,1}}\right)-2 \mathcal{E}
$$

и

$$
\left(H_{03}\left(Z_{j+1,1}\right) \psi_{j, Y_{j+1,1}}, \psi_{j, Y_{j+1,1}}\right) \geqslant \mu\left(\alpha ; Z_{2}\right)\left\|\psi_{j, Y_{j+1,1}}\right\|^{2}, \quad j \geqslant 1,
$$

ибо при $j \geqslant 1$ разбиение $Z_{j+1,1}$ есть разбиение двухкластерной системы $Z_{2}=\left(C_{1}, C_{2}\right)$ не менее чем на 3 кластера за счет дробления кластера $C_{1}$.

3.5. Для оценки в (3.3) члена с $j=0$ мы проводим далее разбиение пространства относительно движения кластера $C_{2}$ в направлении оси $z$ (в ситуации, когда для частиц не разбиваемого кластера $C_{1}$ выполняется $\left.\sum_{t \in C_{1}} q_{t 3}^{2} m_{t} \leqslant b_{1}^{2}\right)$. Для этой цели мы используем функции $u_{Y_{j, 2}}$. Положим

$$
\begin{gathered}
\omega(q)=\psi_{0, Y_{11}}, \quad \widehat{\omega}_{0}=\omega, \quad \widehat{\omega}_{j}=\widehat{\omega}_{j-1}\left(1-\sum_{Y_{j, 2}} u_{Y_{j, 2}}^{2}\right)^{1 / 2}, \\
\omega_{j-1, Y_{j 2}}=\widehat{\omega}_{j-1} u_{Y_{j, 2}}, \quad j=1,2, \ldots, n_{2} \\
\omega_{j}=\sum_{Y_{j 2}} w_{j-1, Y_{j 2}}, \quad j=1,2, \ldots, n_{2}-1
\end{gathered}
$$

и аналогично $(3.3),(3.4)$ получаем

$$
\begin{aligned}
\left(H_{03}\left(Z_{2}\right) \psi_{0, Y_{11}}, \psi_{0, Y_{11}}\right) \geqslant & \sum_{i=0}^{n_{2}-1} \sum_{Z_{i+1,2}}\left(H_{03}\left(Z_{i+1,2}\right) \omega_{i, Y_{i+1,2}}, \omega_{i, Y_{i+1,2}}\right)- \\
& -2 \mathcal{E}\left\|\psi_{0, Z_{11}}\right\|^{2}, \\
\left(H_{03}\left(Z_{i+1,2}\right) \omega_{i, Y_{i+1,2}}, \omega_{i, Y_{i+1,2}}\right) \geqslant & \mu\left(\alpha, Z_{2}\right)\left\|\omega_{i, Y_{i+1,2}}\right\|^{2}, \quad i \geqslant 1 .
\end{aligned}
$$


3.6. Нам остается оценить величину $\left(H_{03}\left(Z_{2}\right) \omega_{0, Y_{12}}, \omega_{0, Y_{12}}\right)$. Для этого разобьем носитель функции $\omega_{0, Y_{12}}$ на области, отвечающие всевозможным распадениям $Z_{s}^{\prime}=$ $\left\{C_{1}^{\prime}, \ldots, C_{s}^{\prime}\right\}$ системы $Z_{1}$ в плоскости $(x, y)$. С этой целью введем сначала пространства

$$
\begin{gathered}
R_{0 \perp}\left(Z_{s}^{\prime}\right)=\left\{q \mid q=\left(q_{1}, \ldots, q_{n}\right), q_{i}=\left(q_{i \perp}, 0\right), q_{i \perp}=\left(q_{i 1}, q_{i 2}\right),\right. \\
\left.\sum_{i \in C_{p}} m_{i} q_{i \perp}=0, p=1,2, \ldots, s\right\}, \\
R_{c \perp}\left(Z_{s}^{\prime}\right)=\left\{q \mid q=\left(q_{1}, \ldots, q_{n}\right), q_{i}=\left(q_{i \perp}, 0\right),\left(q, q^{\prime}\right)_{1}=0 \quad \forall q^{\prime} \in R_{0 \perp}\left(Z_{s}^{\prime}\right)\right\}
\end{gathered}
$$

и обозначим через $P_{\kappa \perp}\left(Z_{s}^{\prime}\right)$ проектор в смысле скалярного произведения $(\cdot, \cdot)_{1}$ на подпространство $R_{\kappa \perp}\left(Z_{s}^{\prime}\right)=0, c, s \geqslant 2$. Пусть, далее, $q_{\perp}=\left(q_{1 \perp}, \ldots, q_{n \perp}\right)$,

$$
\beta_{1}=\left|q_{\perp}\right|_{1}, \quad \beta\left(Z_{s}^{\prime}\right)=\left|P_{0 \perp}\left(Z_{s}^{\prime}\right) q_{\perp}\right|_{1} \cdot\left|P_{c \perp}\left(Z_{s}^{\prime}\right) q_{\perp}\right|_{1}^{-1}, \quad s \geqslant 2 .
$$

Определим числа $\tilde{a}_{s}, \tilde{b}_{s}$ и функции $\tilde{u}_{s}(\beta), \tilde{v}_{s}(\beta)$ аналогично п. 2.4 работы [13] и положим

$$
\begin{gathered}
\tilde{u}_{Z_{s}^{\prime}}=\tilde{u}_{s}\left(\beta\left(Z_{s}^{\prime}\right)\right), \quad \tilde{v}_{Z_{s}^{\prime}}=\tilde{v}_{s}\left(\beta\left(Z_{s}^{\prime}\right)\right), \quad s=1,2, \ldots, n, \quad f=\omega_{0, Y_{12}}, \quad \hat{f}_{0}=f \\
\hat{f}_{i}=f_{i-1}\left(1-\sum_{Z_{i}^{\prime}} \tilde{u}_{Z_{i}^{\prime}}^{2}\right)^{1 / 2}, \quad f_{i-1, Z_{i}^{\prime}}=\hat{f}_{i-1} \tilde{u}_{Z_{i}^{\prime}}, \quad i=1, \ldots, n \\
f_{i}=\sum_{Z_{i}^{\prime}} f_{i-1, Z_{i}^{\prime}}
\end{gathered}
$$

Аналогично предыдушему получаем

$$
\left(H_{03}\left(Z_{2}\right) f, f\right) \geqslant \sum_{j=0}^{n-1} \sum_{Z_{j+1}^{\prime}}\left(H_{03}\left(Z_{2} \cap Z_{j+1}^{\prime}\right) f_{j, Z_{j+1}^{\prime}}, f_{j, Z_{j+1}^{\prime}}\right)-2 \mathcal{E}\|f\|^{2}
$$

где через $Z_{2} \cap Z_{j+1}^{\prime}$ обозначено разбиение $\widetilde{Z}_{p}=\left\{\widetilde{C}_{1}, \ldots, \widetilde{C}_{p}\right\}$, состоящее из тех кластеров $C_{i} \cap C_{k}^{\prime}, C_{i} \in Z_{2}, C_{k}^{\prime} \in Z_{s}^{\prime}$, которые не пусты. Очевидно, при $Z_{s}^{\prime} \neq Z_{1}, Z_{2}$ мы получаем $\widetilde{Z}_{p}<Z_{2}, p \geqslant 3$, и, значит,

$$
\left(H_{03}\left(Z_{2} \cap Z_{j+1}^{\prime}\right) \varphi_{j, Z_{j+1}^{\prime}}, \varphi_{j, Z_{j+1}^{\prime}}\right) \geqslant \mu\left(\alpha, Z_{2}\right)\left\|\varphi_{j, Z_{j+1}^{\prime}}\right\|^{2} \quad \text { при } \quad Z_{j+1}^{\prime} \neq Z_{1}, Z_{2}
$$

При $Z_{j+1}^{\prime}=Z_{1}, Z_{2}$, очевидно, $Z_{2} \cap Z_{j+1}^{\prime}=Z_{2}$. Поэтому в (3.7) нам остается оценить только слагаемые $\left(H_{03}\left(Z_{2}\right) f_{0, Z_{1}}, f_{0, Z_{1}}\right)$ и $\left(H_{03}\left(Z_{2}\right) f_{1, Z_{2}}, f_{1, Z_{2}}\right)$. 
3.7. По построению

$$
f_{0, Z_{1}}(q)=f_{0, Z_{1}}(k, q)=g_{k}(q) u_{11}\left(\tau\left(Y_{11}\right)\right) u_{12}\left(\tau\left(Y_{12}\right)\right) \tilde{u}_{1}\left(\beta_{1}\right),
$$

и, следовательно, при $q \in \operatorname{supp} f_{0, Z_{1}}(k, q)$ для всех $k$ выполняется неравенство

$$
|q|_{1}^{2} \leqslant 2 b_{1}^{2}(1)+\tilde{b}_{1}^{2}(1) .
$$

Спектр оператора $H_{03}\left(\alpha, Z_{2}\right)$ в ограниченной области пространства $R_{03}\left(Z_{2}\right)$ с нулевым граничным условием чисто дискретный с единственной предельной точкой $+\infty$. Поэтому и так как $g_{k}(q)$ сходится слабо к нулю в $\mathcal{L}_{2}\left(R_{03}\left(Z_{2}\right)\right)$, имеем

$$
\underset{k \rightarrow \infty}{\lim }\left(H_{03}\left(Z_{2}\right) f_{0, Z_{1}}(k, q), f_{0, Z_{1}}(k, q)\right) \geqslant \mu\left(\alpha ; Z_{2}\right) \underline{\lim }\left\|f_{0, Z_{1}}(k, q)\right\| .
$$

3.8. Оценим, наконец, величину $\left(H_{03}\left(Z_{2}\right) f_{1, Z_{2}}, f_{1, Z_{2}}\right)$. Предварительно отметим, что по построению при $q \in \operatorname{supp} f_{1, Z_{2}}$

$$
\left|P_{0 \perp}\left(Z_{2}\right) q_{\perp}\right|_{1} \leqslant \tilde{b}_{2}\left|P_{c \perp}\left(Z_{2}\right) q_{\perp}\right|_{1}, \quad\left|q_{\perp}\right|_{1} \geqslant \tilde{a}_{1},
$$

и так как

$$
\left|q_{\perp}\right|_{1}^{2}=\left|P_{0 \perp}\left(Z_{2}\right) q_{\perp}\right|_{1}^{2}+\left|P_{c \perp}\left(Z_{2}\right) q_{\perp}\right|_{1}^{2},
$$

то при $q \in \operatorname{supp} f_{1, Z_{2}}$

$$
\left|P_{c \perp}\left(Z_{2}\right) q_{\perp}\right|_{1} \geqslant \tilde{a}_{1}\left(1+\tilde{b}_{2}^{2}\right)^{-1 / 2} .
$$

Кроме того, в силу леммы 2.3 из [11, с. 125$]$

$$
\begin{aligned}
& \left|P_{c \perp}\left(Z_{2}\right) q_{\perp}\right|_{1}^{2}=\frac{M\left[C_{1}\right] \cdot M\left[C_{2}\right]}{M}\left|\zeta_{\perp}\right|^{2}, \\
& \left|P_{0 \perp}\left(Z_{2}\right) q_{\perp}\right|_{1}^{2} \geqslant\left|q_{i \perp}-\zeta_{\perp}\left[C_{j}\right]\right|^{2} m_{i} \quad \text { при } \quad i \in C_{j},
\end{aligned}
$$

где

$$
\zeta_{\perp}=\zeta_{\perp}\left[C_{1}\right]-\zeta_{\perp}\left[C_{2}\right], \quad \zeta_{\perp}\left[C_{j}\right]=\sum_{t \in C_{j}} m_{t} q_{t \perp} \cdot M\left[C_{j}\right]^{-1} .
$$

После этих простых замечаний оценим снизу в выражении оператора $H_{03}\left(Z_{2}\right)$ член

$$
F=\left(\nu_{1}+\mathcal{E}_{2}\right)^{2}+\left(\nu_{2}-\mathcal{E}_{1}\right)^{2}
$$

где

$$
\mathcal{E}_{j}=2 B \sum_{t=1}^{n} q_{t j} e_{t}
$$

Так как

$$
Q=\sum_{j=1}^{n} e_{j}=0
$$


то

$$
\begin{aligned}
\mathcal{E}_{j}= & 2 B \sum_{s \in C_{1}}\left(q_{s j}-\zeta_{j}\left[C_{1}\right]\right) e_{s}+2 B \sum_{s \in C_{2}}\left(q_{s j}-\zeta_{j}\left[C_{2}\right]\right) e_{s}+ \\
& +2 B \sum_{s \in C_{2}}\left(\zeta_{j}\left[C_{2}\right]-\zeta_{j}\left[C_{1}\right]\right) e_{s}=G_{j}(q)-2 B Q\left[C_{2}\right] \zeta_{j}\left(Z_{2}\right),
\end{aligned}
$$

где в силу (3.9)-(3.12)

$$
\left|G_{j}(q)\right| \leqslant \tilde{b}_{2} c\left|\zeta_{\perp}\left(Z_{2}\right)\right|
$$

и $c$ не зависит от $\tilde{b}_{2}$ и $q$.

В силу (3.13)

$$
\begin{aligned}
F= & \nu_{1}^{2}+\nu_{2}^{2}+2 \nu_{1}\left(G_{2}(q)-2 B Q\left[C_{2}\right] \zeta_{2}\left(Z_{2}\right)\right)- \\
& -2 \nu_{2}\left(G_{1}(q)+2 B Q\left[C_{1}\right] \zeta_{1}\left(Z_{2}\right)\right)+G_{2}^{2}(q)-4 B G_{2}(q) Q\left[C_{2}\right] \zeta_{2}\left(Z_{2}\right)+ \\
& +4 B G_{1}(q) Q\left[C_{1}\right] \zeta_{1}\left(Z_{2}\right)+4 B^{2}\left(Q\left[C_{2}\right]^{2} \zeta_{2}^{2}\left(Z_{2}\right)+Q\left[C_{1}\right]^{2} \zeta_{1}^{2}\left(Z_{2}\right)\right) .
\end{aligned}
$$

Так как число $\tilde{b}_{2}$ может быть выбрано с самого начала достаточно малым, а число $\tilde{a}_{1}-$ достаточно большим, то в силу $(3.14),(3.15)$

$$
F \geqslant 4 B^{2} Q\left[C_{1}\right]^{2}\left|\zeta_{\perp}\right|^{2}-\tilde{b}_{2} c\left|\zeta_{\perp}\right|^{2}-c\left|\zeta_{\perp}\right| \geqslant d_{0}\left|\zeta_{\perp}\right|^{2} \geqslant d_{1} \tilde{a}_{1}^{2}
$$

где константы $d_{0}, d_{1}$ не зависят от $\tilde{a}_{1}$. Следовательно, при достаточно большом $\tilde{a}_{1}$

$$
\left(H_{03}\left(Z_{2}\right) f_{1, Z_{2}}, f_{1, Z_{2}}\right) \geqslant \mu\left(\alpha, Z_{2}\right)\left\|f_{1, Z_{2}}\right\|^{2} .
$$

Собирая вместе оценки (3.3)-(3.8), (3.17), получаем, что

$$
\underline{\lim }\left(H_{03}\left(Z_{2}\right) g_{k}, g_{k}\right) \geqslant \mu\left(\alpha, Z_{2}\right)-\mathcal{E} .
$$

Отсюда и следует утверждение теоремы 1.5.

ЗАМЕЧАНИЕ. Из оценки (3.16) вытекает, что в области $\Omega_{1}=\operatorname{supp} f_{1, Z_{2}}$, отвечаюшей распадению исходной системы на нераспадаюшиеся кластеры $C_{1}, C_{2}$,

$$
\underline{\lim }\left\|g_{k}\right\|_{\Omega_{1}}=O\left(\tilde{a}_{1}^{-1}\right)
$$

где $\left|\tilde{a}_{1}\right| \leqslant$ const $\left|\zeta_{\perp}\left[C_{2}\right]-\zeta_{\perp}\left[C_{1}\right]\right|$ и $\tilde{a}_{1}$ может быть выбрано сколь угодно большим. Таким образом, нами одновременно показано, что последовательность Вейля $g_{k}(q)$ для оператора $H_{03}\left(\alpha ; Z_{2}\right)$ не может отвечать распадению $Z_{2}$ на нераспадающиеся кластеры $C_{1}, C_{2}$. 
3.9. Доказательство теоремы 1.6. Пусть (см. п. 1.4)

$$
\hat{q}_{33}=q_{33}\left(Z_{2}(2)\right)=q_{33}-\sum_{j=1, j \neq 2}^{n} m_{j} q_{j 3} \cdot\left(M-m_{2}\right)^{-1},
$$

$f\left(\hat{q}_{33}\right)$ - гладкая нормированная функция, равная нулю при $\left|\hat{q}_{33}\right| \leqslant 1 ; \hat{q}=q\left(Z_{3}(2,3)\right)$ произвольный вектор из $R_{03}\left(Z_{3}(2,3)\right) ; \varphi(\hat{q})$ - нормированная собственная функция оператора $H_{03}\left(\alpha ; Z_{3}(2,3)\right)$, отвечаюшая его собственному значению $\mu\left(\alpha ; Z_{3}(2,3)\right)$ и обладаюшая перестановочной симметрией типа $\alpha^{\prime \prime}=\alpha^{\prime \prime}\left(Z_{2}(2,3)\right), \alpha^{\prime \prime} \prec \alpha ; \alpha^{\prime}=\alpha^{\prime}\left(Z_{2}(2)\right)$ - такой тип перестановочной симметрии системы $Z_{2}(2)$, что $\alpha^{\prime \prime} \prec \alpha^{\prime} \prec \alpha$. Положим

$$
f_{k}=f\left(k \hat{q}_{33}\right) k^{1 / 2}, \quad \psi_{k}=\varphi f_{k}, \quad \psi_{k}^{\left(\alpha^{\prime}\right)}=P^{\left(\alpha^{\prime}\right)}\left(Z_{2}(2)\right) \psi_{k} .
$$

Подставляя функцию $\psi_{k}^{\left(\alpha^{\prime}\right)}$ в квадратичную форму оператора $H_{03}\left(\alpha ; Z_{3}(2,3)\right)$ и используя известное выражение для оператора $P^{\left(\alpha^{\prime}\right)}$, мы после замены переменных $\tau=k \hat{q}_{33}$ и громоздких выкладок, похожих на проведенные в работе $[14, \S 7]$, получаем, что

$$
\begin{aligned}
\left(H_{03}\left(\alpha, Z_{2}(2)\right) \psi_{k}^{\left(\alpha^{\prime}\right)}, \psi_{k}^{\left(\alpha^{\prime}\right)}\right) & =\left(H_{03}\left(\alpha, Z_{2}(2)\right) \psi_{k}, P^{\left(\alpha^{\prime}\right)} \psi_{k}\right)= \\
& =\mu\left(\alpha ; Z_{2}(2)\right)\left\|\psi_{k}^{\left(\alpha^{\prime}\right)}\right\|^{2}+k(p(k)+O(k)),
\end{aligned}
$$

где при $k \rightarrow 0 \underline{\lim } p(k)<0, \underline{\lim }\left\|\psi_{k}^{\alpha^{\prime}}\right\|^{2}>0$. Поэтому для малых $k$

$$
\inf \left(H_{03}\left(\alpha ; Z_{2}(2)\right) \psi_{k}^{\left(\alpha^{\prime}\right)}, \psi_{k}^{\left(\alpha^{\prime}\right)}\right)<\mu\left(\alpha ; Z_{2}(2)\right)\left\|\psi_{k}^{\left(\alpha^{\prime}\right)}\right\|^{2},
$$

т.e.

$$
\mu^{(\alpha)}:=\inf H_{03}\left(\alpha ; Z_{2}(2)\right) \in \sigma_{\mathrm{d}}\left(H_{03}\left(\alpha ; Z_{2}(2)\right)\right),
$$

что и требовалось доказать.

Благодарности. Работа поддержана грантами РФФИ № 96-01-00478 и INTAS № 95-0414.

\section{Список литературы}

[1] С. А. Вугальтер, Г. М. Жислин. ТМФ. 1997. Т. 113. № 3. С. 413-431.

[2] С. А. Вугальтер. Частное сообщение.

[3] С. А. Вугальтер, Г. М. Жислин. Алгебра и анализ. 1991. Т. 3. № 6. С. 119-155.

[4] С. А. Вугальтер, Г. М. Жислин. Алгебра и анализ. 1993. Т. 5. № 2. С. 108-125.

[5] С. А. Вугальтер, Г. М. Жислин. Тр. Моск. матем. об-ва. 1992. Т. 54. С. 186-212.

[6] В. Я. Иврий. ДАН СССР. 1984. Т. 276. № 2. С. 268-270.

[7] W. Kirsch, B. Simon. Ann. Phys. 1988. V. 183. № 1. P. 122-130.

[8] Ф. Рисс, Б. Секефальви-Надь. Лекции по функциональному анализу. М.: ИЛ, 1954.

[9] Г. М. Жислин. Алгебра и анализ. 1996. Т. 8. № 1. С. 127-136.

[10] J. E. Avron, J. W. Herbst, B. Simon. Ann. Phys. 1978. V. 114. P. 431.

[11] М.А. Антонец, Г.М. Жислин, И.А. Шерешевский. О дискретном спектре многочастичных гамильтонианов. Приложение к кн. К. Йоргенс, И. Вайдманн. Спектральные свойства гамильтоновых операторов. М.: Мир, 1976.

[12] Г. М. Жислин. ТМФ. 1996. Т. 107. № 3. С. 372-387.

[13] С. А. Вугальтер, Г. М. Жислин. ТМФ. 1993. Т. 97. № 1. С. 94-112.

[14] Г. М. Жислин. Изв. АН СССР. Сер. матем. 1969. Т. 33. С. 560-649.

Поступила в редакцию 20.V.1998 г. 